\title{
Stress-Based Topology Optimization of Steel-Frame Structures Using Members with Standard Cross Sections: Gradient-Based Approach
}

\author{
Navid Changizi, S.M.ASCE ; and Mehdi Jalalpour, A.M.ASCE
}

\section{Introduction}

Topology optimization is a free-from design tool that aims to identify optimized material distribution within the design domain. Design performance is quantified with an objective function, which drives the optimization while engineering constraints govern the search domain. Topology optimization offers a systematic search capability of the design space, which allows arriving at revolutionary designs in a variety of engineering applications, especially at the early stages of design conceptualization (Bendsoe and Sigmund 2004). Topology optimization is also increasingly being adopted as a design tool in structural engineering to develop high-performance structural layouts (e.g., Liang et al. 2000; Swan and Rahmatalla 2001; Shea and Smith 2006; Gaynor et al. 2012; Beghini et al. 2014). Structural topology optimization combines mathematical optimization with finite-element analysis to search for optimized member connectivity and sizing under known applied loads and boundary conditions. However, due to difficulties in numerical implementation, structural topology optimization is mainly focused on displacement-based design schemes using truss finite-element models or frames members with relatively simple member crosssectional geometries (e.g., circular). However, there are many applications in structural engineering where stress is the primary design criterion, and thus the recommended designs from an optimization algorithm that is only displacement-based might be suboptimal for controlling the developed stresses within the structure. Moreover, real-world structures may be built with frame structures using members with other cross-sectional shapes (e.g., I-beams). This article focuses on stress-based design of frame structures with standard cross sections using gradient-based design schemes. The challenges for implementing such structural topology optimization as compared with traditional displacement-based truss topology optimization are discussed in the following.

Although truss structures have many applications in structural engineering, they only transfer loads axially. Therefore, if member buckling is not considered, a single design variable (cross-sectional area of members) is adequate to formally express structural performance. However, frame structures are capable of transferring loads with axial, shear, and bending load mechanisms. Therefore moment of inertia also plays an important role in quantifying structural performance and needs to be incorporated into the design process. For simple cross-sectional shapes, such as circular or rectangular sections, an analytical equation can be derived for relating cross-sectional area to moment of inertia. Structures using these cross-sectional shapes for members were used for design under local and global instability constraints (Achtziger 1999; Ohsaki and Katoh 2005; Richardson et al. 2012; Torii et al. 2015), optimizing vehicle bodies (Fredricson et al. 2003; Pedersen 2003, 2004), material selection and cross-section design (Fredricson 2005; Takezawa et al. 2007), and reliabilitybased design (Mogami et al. 2006), using displacement-based objectives. However, actual structures are usually designed from an available library of standard sections (for example, factorybuilt I-beams). Using discrete topology optimization schemes, the available library of candidate sections can be searched and the member cross-sectional areas determined for optimal truss designs (Achtziger and Stolpe 2007; Rasmussen and Stolpe 2008; Kanno and Guo 2010; Stolpe 2015) and moment of inertia for an optimal frame design (Kureta and Kanno 2014). However, the computational cost of these topology optimization methods increases rapidly with increasing the number of available candidates. In general, gradient-based optimizations are recommended for topology optimization where the number of design variables is relatively large (Sigmund 2011). However, analytical equations for expressing moment of inertia for such cross sections do 
not exist, which prohibits using these efficient gradient-based optimizers.

With regards to effectively solving stress-based topology optimization problems, there are significant challenges that need to be addressed. The first is the so-called singularity phenomenon, the second is related to the highly nonlinear stress behavior, and the third is related to challenges with adequately expressing structural performance with a scalar that represents stress variation within the domain (Rozvany 2001; Cheng and Guo 1997; Cheng and Jiang 1992). The singularity problem was first encountered for truss designs subject to stress constraints where it was shown that the n-dimensional feasible design space contains degenerate subspaces of dimensions less than $n$ (Cheng and Jiang 1992; Kirsch 1990), where the optimal design often laid in these degenerate subspaces. Nonlinear programming algorithms cannot identify these regions and hence they converge to locally optimized designs. To remedy this situation, several relaxation approaches have been proposed for truss design [e.g., the $\varepsilon$-relaxation and smooth envelope functions (Cheng and Guo 1997; Rozvany and Sobieszczanski-Sobieski 1992)]. These approaches were later adopted for the stressconstrained design of continua (Duysinx and Bendsøe 1998; Bruggi 2008). Another issue with stress constraints, which is more pronounced in continua, is their highly nonlinear dependence on the design. Because stress is directly dependent on the density, it is significantly affected by density changes in neighboring regions (especially if a region with very small densities is being formed), which poses numerical instabilities for optimizers. The final difficulty of stress-based topology optimization is due to the local nature of the stress constraint. Stress changes within the design domain, and constraining stress in every point of the structure is computationally prohibitive for even moderate-sized topology optimization problems. Alternatively, stresses can be evaluated at many points within the domain, and the maximum of these (for example) could be controlled, which poses additional computational time. In addition, the maximum is not a differentiable function, which again precludes application of efficient gradient-based optimizers. A proposed solution to this complication replaces the local stress constraints with a single integrated stress constraint that approximates the maximum stress, such as the $p$-norm or the KreisselmeierSteinhauser (KS) functions (Yang and Chen 1996; Duysinx and Sigmund 1998; Le et al. 2010), which is adopted in this work and recent papers concerned with stress-based design under uncertainty (e.g., Changizi et al. 2017).

This paper proposes an efficient methodology for stress-based topology optimization of frames with structural member properties that are mapped from an available library of candidate cross sections. To more accurately account for the natural variability of the data pertaining to cross-sectional properties, quantile regression is employed to analytically express these properties in terms of member cross-sectional area, which is the design variable. Specifically, the American Institute of Steel Construction (AISC) design manual is used for member selection using I-beam members. In this paper, I-beams refers to the collection of sections under HP, S, M, and $\mathrm{W}$ in the AISC design manual (AISC 2015). Moreover, stressbased performance of the structure is directly incorporated in the design objective using the maximum of the von Mises yield criterion throughout the domain, where the maximum is approximated with a differentiable $p$-norm function. These strategies allowed employing efficient gradient-based optimizers for searching the design domain for optimized designs. Three numerical examples were optimized with the proposed methodology to verify its effectiveness. Moreover, these designs were compared with traditional compliance-based designs. A methodology to interpret these designs as actual cross sections of the AISC design manual is also presented, and is shown to lead to minimal changes in structural performance.

\section{Compliance-Based Topology Optimization of Frame Structures}

This section begins by reviewing the compliance-based topology optimization, which can be defined as follows:

$$
\begin{gathered}
\underset{\mathbf{a}}{\min } C=\mathbf{f}^{T} \mathbf{d}(\mathbf{a}) \\
\text { s.t. } \quad \mathbf{K}(\mathbf{a}) \mathbf{d}(\mathbf{a})=\mathbf{f} \\
\mathbf{a}^{T} \mathbf{l} \leq v \\
a_{\min }<a \leq a_{\max }
\end{gathered}
$$

Throughout this article, boldface lowercase and uppercase letters symbolize vectors and matrices, respectively. In the above formulation, $C$ is the compliance, $\mathbf{f}$ is the force vector, and $\mathbf{l}$ is the vector of element lengths. Total amount of available material is denoted by $v$. Moreover, $\mathbf{K}$ and $\mathbf{d}$ are, respectively, the global stiffness matrix and displacement vector, obtained from finite element analysis. The design variables are member cross-sectional areas stored in the vector $\mathbf{a}$, with an individual member of $a ; a_{\min }$ is a small quantity for cross-sectional area to avoid singularity of the stiffness matrix, and $a_{\max }$ is the maximum allowable cross-sectional area.

As discussed previously, because frame structures consist of members capable of transferring axial and shear forces as well as bending moment, member moment of inertia $(I)$ in addition to cross-sectional area $(a)$ contributes to the element-level stiffness matrix and thus becomes important in topology optimization. To avoid treating moment of inertia as a new design variable, it should be expressed in terms of member cross-sectional area. Although such expressions are straightforward to derive for simple crosssectional geometries (for instance, for circular cross sections $I=1 / 4 \pi a^{2}$ ), for I-beams which are mainly used for construction of many frame structures (due to their bending efficiency), an equation relating $I$ to $a$ does not exist. As a first-step approximation, a simple relationship $I=\beta a^{2}$ was assumed (Changizi and Jalalpour 2017) for compliance-based design of frames under uncertainty, where $\beta$ related bending stiffness to axial stiffness (bending efficiency of members). The range for this coefficient was determined using the AISC manual (AISC 2015). However, because the focus of the present paper is on stress-based topology optimization, other cross-sectional properties should be expressed in terms of cross-sectional area as well. Therefore, the use of a variant of regression models to establish a relationship between member cross-sectional area, which is the design variable, and moment of inertia and other cross sectional properties (as needed throughout this paper) is proposed. The application is restricted to the AISC design manual data for I-beams. Therefore $a_{\max }$ in this work will be the upper bound of area given by the AISC design manual.

\section{Establishing Relationship between Area and Other Cross-Sectional Properties}

The data for I-beam sections from the AISC manual are shown in Fig. 1 for two of the section properties, moment of inertia $I$ and 

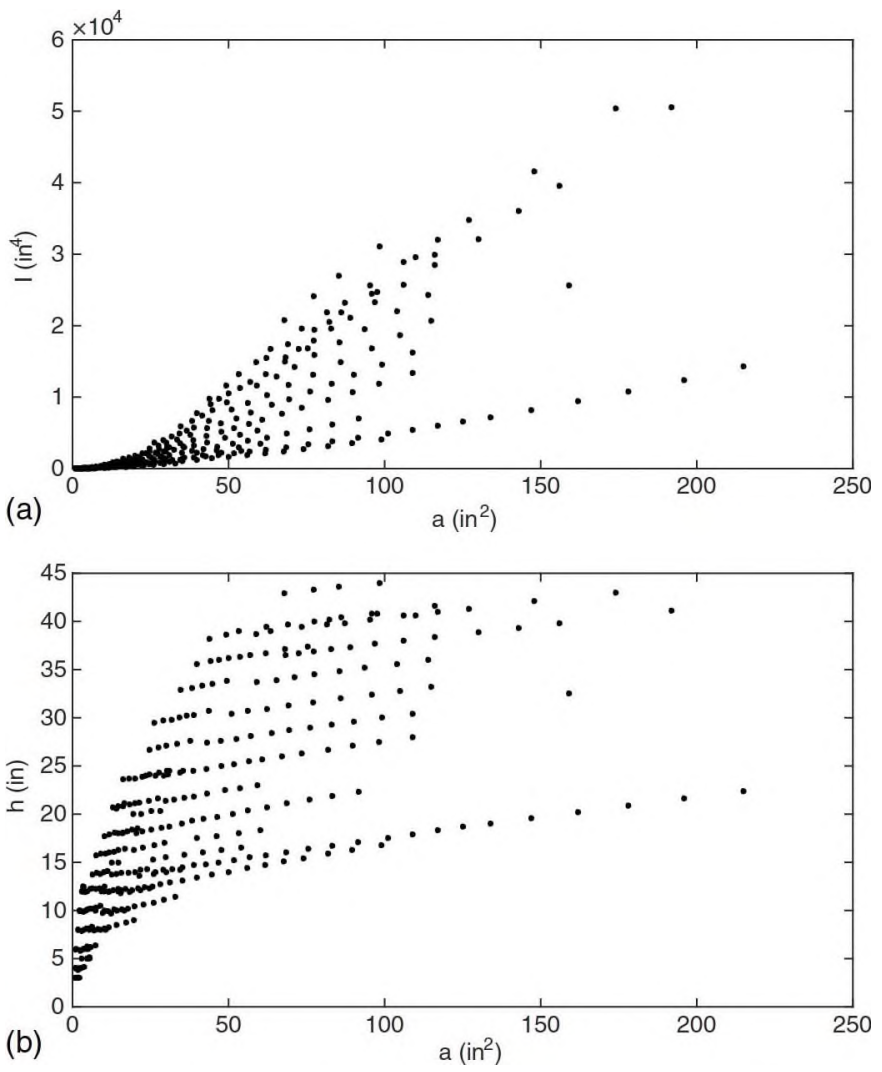

Fig. 1. Section properties versus cross-sectional area for the I-beams in the AISC design manual: (a) moment of inertia; (b) section depth

section depth $h$, against cross-sectional area $a$. There is a total of 340 data points.

A first choice for establishing a relationship between crosssectional area and other section properties is the use of linear regression models. These models provide an equation relating the average of the moment of inertia (for example) given the observed cross-sectional area. This methodology was used for a limited data set of W-sections in Sarma and Adeli (2000). In regression analysis, it is preferred to use a model with a minimum number of parameters to control the standard error of the estimated parameters while maintaining acceptable prediction accuracy [see Amini et al. (2016) and references therein for more discussion of this]. Moreover, this paper requires that model predictions are always positive, and are zero if cross-sectional area (the predictor) is zero. Therefore the square root of moment of inertia is predicted using the following model (without an intercept term):

$$
v^{\prime} \bar{I}=\alpha_{1} a+\alpha_{2} a^{2}+\alpha_{3} a^{3}
$$

where $\alpha$ coefficients are estimated from the data. However, it is observed that the current data are greatly dispersed and a single model to predict the average moment of inertia given $a$ would not be sufficient. Therefore it is proposed to employ quantile regression for this data set. Unlike ordinary regression, which yields one model for the conditional mean of the response variable $(I$, for example) given predictors ( $a$, in this case), quantile regression aims at estimating either the conditional median ( 0.5 quantile or 50 th percentile) or other quantiles of the response variable (Koenker and Bassett Jr 1978). Therefore, quantile regression offers the flexibility to use a distinct model for each representative percentile of the data. In this paper, three sets of models between area and other cross-sectional properties are established for the 10th, 50th, and 90th percentiles. These percentiles were chosen to represent sections with low, medium, and high bending efficiency, respectively, as shown in Fig. 2. In this figure, the cross-section of each I-beam representing the maximum point of each chosen percentile is drawn to scale to clearly show section geometry. From a pure bending-capacity design perspective, sections similar to the one shown on the right side of Fig. 2 are preferred, because with a lower cross-sectional area (the design variable in this work), a higher moment of inertia can be achieved (high bending efficiency).

The results from quantile regression are presented in Appendix I. These equations are needed to perform a compliance-based design of frames using sections from the AISC design manual via the problem formulation in Eq. (1). The only change compared with design with simpler cross-sections (circular, for example) is that once the desired percentile is chosen, Eq. (2) can be used to determine moment of inertia for finite-element stiffness matrices as a function of cross-sectional area. The design variables are still the crosssectional areas. In the Numerical Examples section, compliancebased designs with each of these three percentiles are presented and changes to the topology are discussed. In principle, each member could be chosen from a separate percentile. For instance, in practical situations beam members could be chosen from upper percentiles and bracing members could be chosen from lower percentiles. Such an implementation of the proposed methodology is also straightforward and only requires additional bookkeeping. For stress-based design, additional equations for other section properties, such as $h$ (section depth), are needed. Models with the following structures for predicting these cross-sectional properties are used:

$$
h=\beta_{1} \sqrt{a}+\beta_{2} a+\beta_{3} \sqrt[2]{a^{3}}
$$

In Eq. (3) model coefficients $(\beta)$ are also estimated from the data. Similar equations for flange width $b_{f}$ and thickness $t_{f}$ and web thickness $t_{w}$ were used. However, it can be seen in Fig. 2 that cross sections at the lowest percentile of the moment of inertia do not necessarily have the lowest flange and web thickness. Observation of the data suggests using $90 \%$ equations for the aforementioned properties in this data set. Model predictions were verified for the numerical examples in this paper, and a very good agreement was observed.

\section{Stress-Based Topology Optimization of Frame Structures}

Unlike compliance-based design, which uses the global vector of displacements to compute the objective function, stress-based design requires computing internal forces in frame members to calculate stresses within the domain. Moreover, a stress criterion that combines several stresses (normal and shear stresses) in a point into a single scalar is needed. In addition, because stress changes within the domain (along the length of elements and depth of crosssection), and computing stress in every point of the structure is computationally demanding, the stress criterion should be evaluated in a finite number of points. Finally, these scalar numbers should be combined into a single objective or a few constraints to avoid problems with very large numbers of constraints or objective functions. The strategies for addressing these challenges are presented in the following sections.

\section{von Mises Yield Criterion}

The von Mises yield criterion is shown to be accurate for predicting the onset of yielding of metals, and therefore is used in this work to 


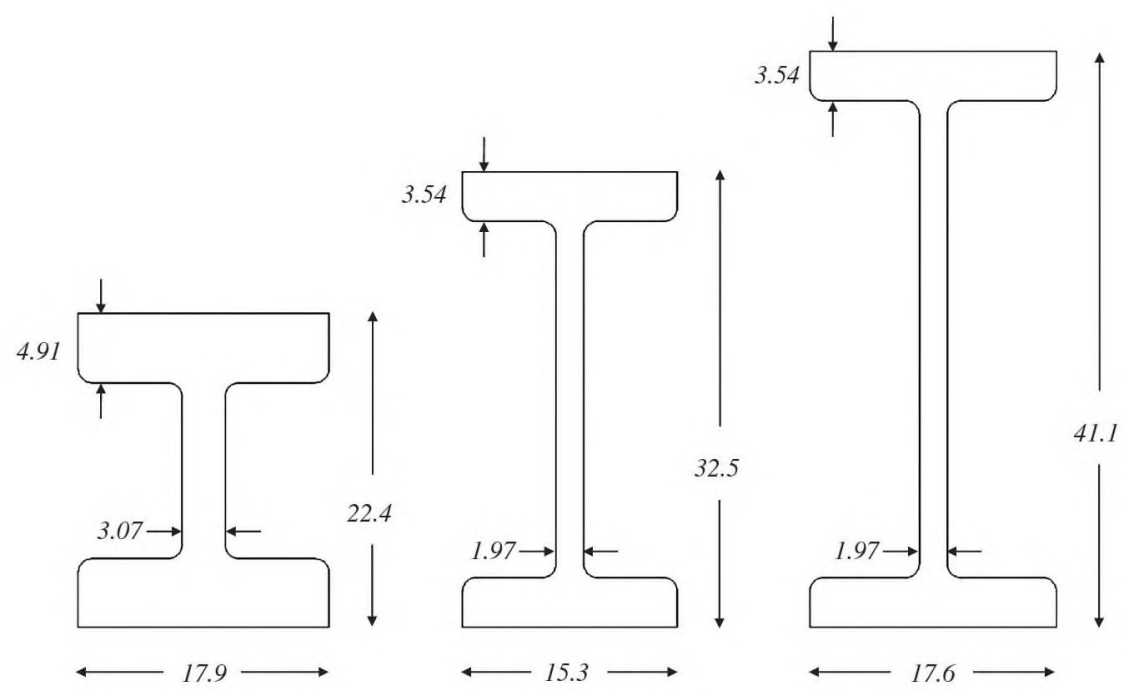

Fig. 2. I-beam cross sections from the AISC design manual with maximum area in each of the chosen percentiles: $10 \%$ (left, $a=215$, $I=1.43 \times 10^{4}$ ), $50 \%$ (middle, $a=159, I=2.56 \times 10^{4}$ ), and $90 \%$ (right, $a=192, I=5.06 \times 10^{4}$ )

measure the structural performance. The general form of the von Mises yield criterion is

$$
S=\sqrt{\boldsymbol{\sigma}^{T} \mathbf{U} \boldsymbol{\sigma}}
$$

where $S=$ von Mises yield criterion at a point; $\boldsymbol{\sigma}=$ vector that collects all stresses developed in that point; and $\mathbf{U}=$ matrix of coefficients. Under plane stress conditions, the matrix of coefficients $\mathbf{U}$ is expressed as

$$
\mathbf{U}=\left[\begin{array}{ccc}
1 & -0.5 & 0 \\
-0.5 & 1 & 0 \\
0 & 0 & 3
\end{array}\right]
$$

For planar frames, the stress vector $\sigma$ is

$$
\boldsymbol{\sigma}^{T}=\left\{\begin{array}{lll}
\sigma_{11} & \sigma_{22} & \sigma_{12}
\end{array}\right\}
$$

where $\sigma_{11}, \sigma_{22}$, and $\sigma_{12}=$ nomal and shear stresses. Next. computation of these stresses within the domain is discussed.

\section{Determining Stress-Based Performance of Frames}

To compute the stresses that are developed within the structure members under the application of external forces $\mathbf{f}$, internal forces at both ends of each frame member are computed first using

$$
\mathbf{f}_{e l}=\mathbf{K}_{e l} \mathbf{T d}
$$

where $\mathbf{f}_{e l}, \mathbf{K}_{e l}, \mathbf{T}$, and $\mathbf{d}=$ internal forces for each element, local stiffness matrix, transformation matrix, and the displacement vector, respectively, obtained from finite-element analysis. Next, internal forces in any location of a specific member are calculated and are used to determine normal and shear stresses as follows (under plane stress conditions):

$$
\begin{gathered}
\sigma_{11}= \pm \frac{M y}{I}-\frac{P}{a} \\
\sigma_{22}=0 \\
\sigma_{12}=\frac{V Q}{I t}
\end{gathered}
$$

where $M, V$, and $P=$ internal bending moment, shear force, and axial force respectively; $y=$ distance away from neutral axis of the

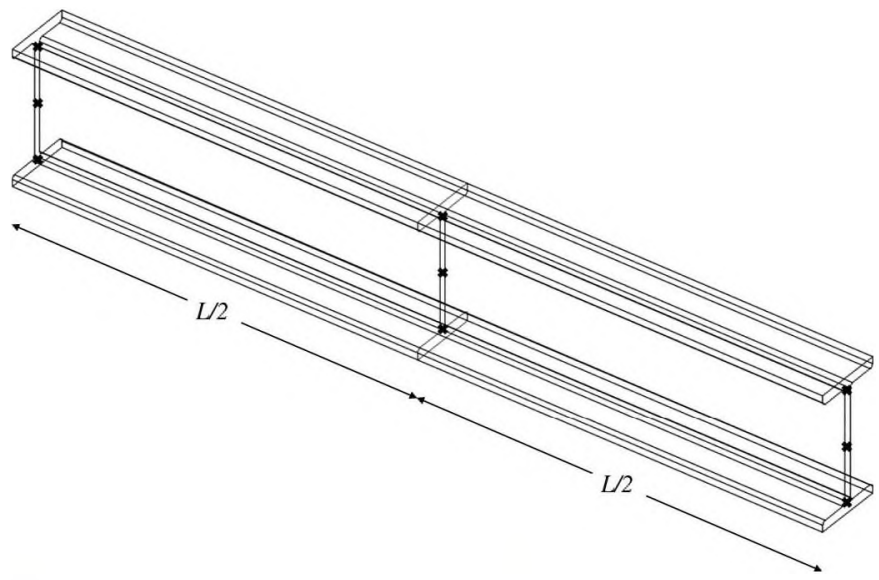

Fig. 3. Location of the proposed nine points for computing von Mises stress in each frame element

cross section; $a=$ cross-sectional area; $I=$ moment of inertia; $Q=$ first moment of area; and $t=$ width at the section where shear stress is being evaluated. Therefore, for stress calculations for planar frames, two coordinates are required: (1) the location $x$ on the length of a member; and (2) the distance $y$ on each cross-section from the neutral axis. In this paper stresses are evaluated in nine points for each frame member as shown in Fig. 3. These nine points are the points most susceptible to generating the maximum combined stress (measured in terms of the von Mises criterion) for each member. However, the number of points can be decreased based on type and location of loading (for example, point loads applied on connections only).

For simplicity of calculations, a vector and a matrix to collect the required forces along the axis of the member $(x)$ and the coefficients representing the location on the cross section $(y)$ are defined. Multiplication of these yields the stress vector in a given point as follows:

$$
\boldsymbol{\sigma}=\mathbf{R}_{v} \mathbf{q}_{x}
$$

In Eq. (9), $\mathbf{q}_{x}$ extracts the required forces in the $x$-direction and matrix $\mathbf{R}_{v}$ is related to section properties and the location on the cross section. The vector $\mathbf{q}_{\mathrm{r}}$ is defined as follows: 


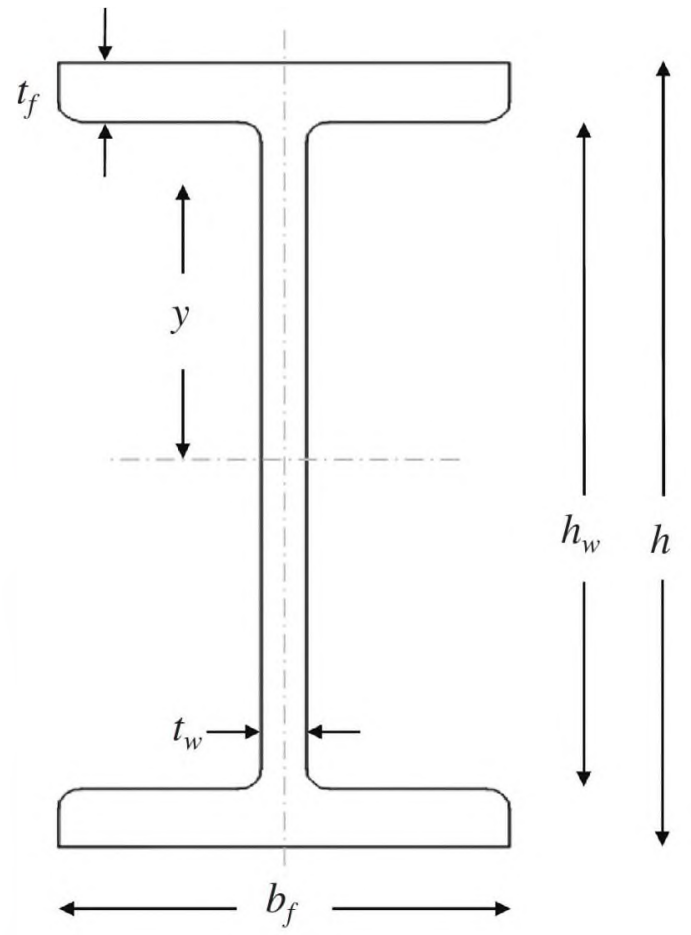

Fig. 4. Section parameters for an I-beam

$$
\mathbf{q}_{x}^{T}=\{P \quad V \quad M\}
$$

As shown in Fig. 3, $x=[0, L / 2, L]$ is chosen in this paper, where $L$ is member length. Having determined the required forces, attention is now turned to the coordinate on the cross section. A typical I-beam cross section is shown in Fig. 4. The matrix $\mathbf{R}_{v}$ for location along the cross-section is defined in general as follows:

$$
\mathbf{R}_{y}=\left[\begin{array}{ccc}
c_{a} & 0 & c_{b} \\
0 & 0 & 0 \\
0 & c_{s} & 0
\end{array}\right]
$$

where $y$ denotes the position measured from the neutral axis of the section as shown in Fig. 4. There are three coefficients in Eq. (11): axial stress coefficient $c_{a}$; bending stress coefficient $c_{b}$; and shear stress coefficient $c_{s}$. These coefficients depend on the shape of the cross section and the location where stress is being evaluated. For I-beams, in general, these coefficients are

$$
\begin{gathered}
c_{a}=\frac{-1}{a} \\
c_{b}=\frac{y}{I} \\
c_{s}=\frac{b_{f}\left(h^{2}-h_{w}^{2}\right)}{8 t_{w} I}+\frac{\frac{t_{w}}{2}\left(\frac{h_{w}^{2}}{4}-y^{2}\right)}{t_{w} I}
\end{gathered}
$$

The parameters in Eq. (12) are shown in Fig. 4.

The axial stress coefficient is constant for all locations on the cross section, whereas the other coefficients vary. As shown in Fig. 3, the stresses are determined at three points along the cross-section: $y=h / 2-t_{f}, y=0$, and $y=-h / 2+t_{f}$. Therefore the stress coefficients used in Eq. (11) can be determined specifically. First, for $y=h / 2-t_{f}$ the following is derived:

$$
\begin{gathered}
c_{b}=\frac{\frac{h}{2}-t_{f}}{I} \\
c_{s, \min }=\frac{b_{f}\left(h^{2}-h_{w}^{2}\right)}{8 t_{w} I}
\end{gathered}
$$

For $y=0$

$$
\begin{gathered}
c_{b}=0 \\
c_{s, \max }=\frac{b_{f}\left(h^{2}-h_{w}^{2}\right)+t_{w} h_{w}^{2}}{8 t_{w} I}
\end{gathered}
$$

Finally, for $y=-\frac{h}{2}+t_{f}$

$$
\begin{gathered}
c_{b}=\frac{\frac{-h}{2}+t_{f}}{I} \\
c_{s, \min }=\frac{b_{f}\left(h^{2}-h_{w}^{2}\right)}{8 t_{w} I}
\end{gathered}
$$

As mentioned previously, multiplication of $\mathbf{R}_{y}$ and $\mathbf{q}_{x}$ yields the stress vector at each point on the frame element. The von Mises yield criterion can now be determined by rewriting Eq. (4) as

$$
S=\sqrt{\left(\mathbf{R}_{y} \mathbf{q}_{x}\right)^{T} \mathbf{U}\left(\mathbf{R}_{y} \mathbf{q}_{x}\right)}
$$

Returning to Fig. 3 and assuming the frame consists of $m$ members, the criterion is evaluated at $m \times 9$ points within the domain at each optimization iteration. Note that depending on the application, stress could be evaluated at other candidate points as well. The goal of this optimization is to control the maximum of these stresses within the design domain. However, because the maximum is not a differentiable function, it is approximated with the p-norm function as $\max \left(S_{\alpha \beta}\right) \approx\left(\sum_{\alpha=1}^{m} \sum_{\beta=1}^{9} S_{\alpha \beta}^{p}\right)^{1 / p}$ (Yang and Chen 1996; Duysinx and Sigmund 1998; Le et al. 2010). The larger the exponent $p$, the closer the approximation.

\section{Stress-Based Topology Optimization Problem Definition and Sensitivities}

Putting the preceding discussions together, the proposed stressbased topology optimization is defined as follows:

$$
\begin{gathered}
\min _{\mathbf{a}} S=\left(\sum_{\alpha=1}^{m} \sum_{\beta=1}^{9} S_{\alpha \beta}^{p}\right)^{1 / p} \\
\text { s.t. } \quad \mathbf{K}(\mathbf{a}) \mathbf{d}(\mathbf{a})=\mathbf{f} \\
\mathbf{a}^{T} \mathbf{l} \leq v \\
a_{\min }<a \leq a_{\max }
\end{gathered}
$$

The major change with respect to Eq. (1) is the objective function $S$, which is the $p$-norm of all stresses with a sufficiently large norm to approximate the maximum. Because of the large number of design variables in frame stress-based topology optimization, it is desirable to use gradient-based optimizers to reduce the computational cost (Sigmund 2011). The sensitivity for the proposed objective function is derived using direct differentiation method in what follows.

Differentiating the objective function with respect to a design variable ( $a_{e}$, where subscript $e$ is dropped for brevity) yields 


$$
\frac{\partial S}{\partial a}=\frac{1}{p}\left(\sum_{\alpha=1}^{N e} \sum_{\beta=1}^{9} S_{\alpha \beta}^{p}\right)^{(1-p) / p} \frac{\partial\left(\sum_{\alpha=1}^{N e} \sum_{\beta=1}^{9} S_{\alpha \beta}^{p}\right)}{\partial a}
$$

Eq. (21) is composed of two components. Because calculation of the first component is straightforward, the second component is focused on, which can be expressed as

$$
\frac{\partial\left(\sum_{i=1}^{n} S_{\alpha \beta}^{p}\right)}{\partial a}=\sum_{\alpha=1}^{N e} \sum_{\beta=1}^{9} \frac{\partial S_{\alpha \beta}^{p}}{\partial a}=\sum_{\alpha=1}^{N e} \sum_{\beta=1}^{9} S_{\alpha \beta}^{p-1} \frac{\partial S_{\alpha \beta}}{\partial a}
$$

Therefore the derivative of each computed von Mises criterion with respect to a design variable is needed, which by differentiating $\mathrm{Eq}$. (19) is given as

$$
\begin{aligned}
\frac{\partial S_{\alpha \beta}}{\partial a}= & \left(\frac{\partial \mathbf{q}_{x}^{T}}{\partial a} \mathbf{R}_{y}^{T} \mathbf{V} \mathbf{R}_{y} \mathbf{q}_{x}+\mathbf{q}_{x}^{T} \frac{\partial \mathbf{R}_{y}^{T}}{\partial a} \mathbf{U} \mathbf{R}_{y} \mathbf{q}_{x}\right. \\
& \left.+\mathbf{q}_{x}^{T} \mathbf{R}_{y}^{T} \mathbf{U} \frac{\partial \mathbf{R}_{y}}{\partial a} \mathbf{q}_{x}+\mathbf{q}_{x}^{T} \mathbf{R}_{y}^{T} \mathbf{U} \mathbf{R}_{y} \frac{\partial \mathbf{q}_{x}}{\partial a}\right) / 2 S_{\alpha \beta}
\end{aligned}
$$

The derivatives $\partial \mathbf{R}_{y} / \partial a$ and $\partial \mathbf{q}_{x}^{T} / \partial a$ are determined next. Focusing on $\partial \mathbf{R}_{y} / \partial a$, it is found that

$$
\frac{\partial \mathbf{R}_{y}}{\partial a}=\left[\begin{array}{ccc}
\frac{\partial c_{a}}{\partial a} & 0 & \frac{\partial c_{b}}{\partial a} \\
0 & 0 & 0 \\
0 & \frac{\partial c_{s}}{\partial a} & 0
\end{array}\right]
$$

with entries

$$
\begin{gathered}
\frac{\partial c_{a}}{\partial a}=\frac{1}{a^{2}} \\
\frac{\partial c_{b}}{\partial a}=\frac{\left(\frac{\partial h}{2 \partial a}-\frac{\partial t_{f}}{\partial a}\right)}{I}-\frac{\frac{\partial I}{\partial a}\left(\frac{h}{2}-t_{f}\right)}{I^{2}}
\end{gathered}
$$

For the shear coefficient $c_{s}$, the derivatives of maximum and minimum are determined separately as follows:

$$
\begin{aligned}
\frac{\partial c_{s, \max }}{\partial a}= & \left\{\left[2 \frac{\partial b_{f}}{\partial a}\left(h^{2}-h_{w}^{2}\right)+b_{f}\left(2 \frac{\partial h}{\partial a} h-2 \frac{\partial h_{w}}{\partial a} h_{w}\right)\right.\right. \\
& \left.+\frac{\partial t_{w}}{\partial a} h_{w}^{2}+2 t_{w} h_{w} \frac{\partial h_{w}}{\partial a}\right] 8 t_{w} I-8\left(\frac{\partial t_{w}}{\partial a} I+\frac{\partial I}{\partial a} t_{w}\right) \\
& \left.\times\left[b_{f}\left(h^{2}-h_{w}^{2}\right)+t_{w} h_{w}^{2}\right]\right\} /\left(64 t_{w}^{2} I^{2}\right) \\
\frac{\partial c_{s, \min }}{\partial a}= & \left\{\left[2 \frac{\partial b_{f}}{\partial a}\left(h^{2}-h_{w}^{2}\right)+b_{f}\left(2 \frac{\partial h}{\partial a} h-2 \frac{\partial h_{w}}{\partial a} h_{w}\right)\right] 8 t_{w} I\right. \\
& \left.-8\left(\frac{\partial t_{w}}{\partial a} I+\frac{\partial I}{\partial a} t_{w}\right)\left[b_{f}\left(h^{2}-h_{w}^{2}\right)\right]\right\} /\left(64 t_{w}^{2} I^{2}\right)
\end{aligned}
$$

It can be seen that derivatives of cross-sectional properties with respect to $a$ are required. Therefore, by differentiating Eq. (2), the following is obtained:

$$
\frac{\partial I}{\partial a}=2 \sqrt{I}\left(\alpha_{1}+2 \alpha_{2}+3 \alpha_{3} a^{2}\right)
$$

For brevity, the derivative of $h$ is only presented by differentiating Eq. (3) as follows:

$$
\frac{\partial h}{\partial a}=\beta_{1} \frac{1}{2 \sqrt{a}}+\beta_{2}+\beta_{3} \frac{3 \sqrt{a}}{2}
$$

Derivatives of other cross-sectional properties are similar. Next, $\partial \mathbf{q}_{x}^{T} / \partial a$ is expressed as

$$
\frac{\partial \mathbf{q}_{x}^{T}}{\partial a}=\left\{\begin{array}{lll}
\frac{\partial P}{\partial a} & \frac{\partial V}{\partial a} & \frac{\partial M}{\partial a}
\end{array}\right\}
$$

which translates into determining the gradient of internal forces. These can be categorized into two sets: element $e$ and other elements. The derivatives of internal forees for element $e$ are given as

$$
\frac{\partial \mathbf{f}_{e l}}{\partial a}=\frac{\partial \mathbf{K}_{e l}}{\partial a} \mathbf{T d}+\mathbf{K}_{e l} \mathbf{T} \frac{\partial \mathbf{d}}{\partial a}
$$

For frame elements other than element $e$, only the second term of Eq. (31) exists. Using direct differentiation, derivatives of element local stiffness matrix are

$$
\frac{\partial \mathbf{K}_{e l}}{\partial a}=\frac{E}{L}\left[\begin{array}{cccccc}
1 & -1 & 0 & 0 & 0 & 0 \\
-1 & 1 & 0 & 0 & 0 & 0 \\
0 & 0 & \frac{12 \frac{\partial I}{\partial a}}{L^{2}} & \frac{-12 \frac{\partial I}{\partial a}}{L^{2}} & \frac{6 \frac{\partial I}{\partial a}}{L} & \frac{-6 \frac{\partial I}{\partial a}}{L} \\
0 & 0 & \frac{-12 \frac{\partial I}{\partial a}}{L^{2}} & \frac{12 \frac{\partial I}{\partial a}}{L^{2}} & \frac{-6 \frac{\partial I}{\partial a}}{L} & \frac{6 \frac{\partial I}{\partial a}}{L} \\
0 & 0 & \frac{6 \frac{\partial I}{\partial a}}{L^{2}} & \frac{-6 \frac{\partial I}{\partial a}}{L^{2}} & \frac{4 \frac{\partial I}{\partial a}}{L} & \frac{2 \frac{\partial I}{\partial a}}{L} \\
0 & 0 & \frac{6 \frac{\partial I}{\partial a}}{L^{2}} & \frac{-6 \frac{\partial I}{\partial a}}{L^{2}} & \frac{2 \frac{\partial I}{\partial a}}{L} & \frac{4 \frac{\partial I}{\partial a}}{L}
\end{array}\right]
$$

The derivative for the displacement vector is also determined as

$$
\frac{\partial \mathbf{d}}{\partial a}=-\mathbf{K}^{-1} \frac{\partial \mathbf{K}}{\partial a} \mathbf{d}
$$

Substituting Eq. (33) into Eq. (31) yields

$$
\frac{\partial \mathbf{f}_{e l}}{\partial a}=\frac{\partial \mathbf{K}_{e l}}{\partial a} \mathbf{T d}-\mathbf{K}_{e l} \mathbf{T} \mathbf{K}^{-1} \frac{\partial \mathbf{K}}{\partial a} \mathbf{d}
$$

To avoid computing the inverse of the stiffness matrix, the second part of Eq. (34) can be written as

$$
\mathbf{K}_{e l} \mathbf{T K}^{-1} \frac{\partial \mathbf{K}}{\partial a} \mathbf{d}=\mathbf{K}_{e l} \mathbf{T K}^{-1} \mathbf{o}
$$

Therefore the solution to the following linear problem is required:

$$
\mathbf{o}=\mathbf{K} \xi
$$

And so, in place of the second term in Eq. (34), the following is computed:

$$
\mathbf{K}_{e l} \mathbf{T K}^{-1} \frac{\partial \mathbf{K}}{\partial a} \mathbf{d}=\mathbf{K}_{e l} \mathbf{T} \boldsymbol{\xi}
$$

which leads to

$$
\frac{\partial \mathbf{f}_{e l}}{\partial a}=\frac{\partial \mathbf{K}_{e l}}{\partial a} \mathbf{T d}-\mathbf{K}_{e l} \mathbf{T} \boldsymbol{\xi}
$$

Solving the optimization problem for the vector of element design variables a requires several steps, which are given below.

1. Choose a percentile for moment of inertia and perform quantile regression analysis to determine the required regression coefficients. For selected percentiles of all I-beam sections in the AISC design manual, refer to the results of Appendix I. Set $a_{\max }$ to the maximum allowable area according to the chosen 
percentile. Note that multiple percentiles could be chosen for different members of the design.

2. Begin the optimization procedure with an initial guess for the vector of design variables a.

3. Solve the equilibrium equation for $\mathbf{d}$ with finite element analysis.

4. Compute the von Mises stresses in nine points for each element of structure using Eq. (19).

5. Evaluate the $p$-norm of all calculated stresses to find the maximum using Eq. (20).

6. Compute the sensitivity of the objective function with respect to design variables as follows:

a. Determine the sensitivity of internal forces using Eq. (38) with the help of Eqs. (30)-(37).

b. Compute entries of matrix $\mathbf{R}_{v}$ for all points using Eqs. (24)-(27) with the help of Eqs. (28) and (29).

c. Compute the sensitivity using Eq. (21) with the help of Eqs. (22) and (23).

7. Update the design variable vector a, using a gradient-based optimizer.

8. Update other section properties, such as $I$ and $h$, using equations resulted from quantile regression.

9. Check convergence; if not converged, return to Step (2), otherwise the solution is deemed final.

The solutions in this paper were found using a uniform material distribution as the initial guess (equal area for all members), and using the MATLAB optimization toolbox (with the fmincon function using the interior point method) as the optimizer (MATLAB). The optimizer tolerance was chosen as $0.0001 \%$. In addition, the MMA algorithm of Svanberg (1987) was used as the optimizer, and it was found that the MATLAB optimizer performed slightly better for these examples; this observation is in line with Rojas-Labanda and Stolpe (2015). Note that convergence to the global minimum is not guaranteed.

\section{Numerical Examples}

In this section, the proposed algorithm is used for stress-based design of three frame structures, and results are compared with traditional compliance-based designs. All frames incorporate I-beam members from the AISC design manual. To study the effects of bending efficiency of members on the final topology, each frame was designed at three percentile levels, 10,50 , and $90 \%$, for moment of inertia (Fig. 2). The ground structure approach was used, where a dense mesh of candidate elements was used to initialize the design process. The proposed algorithm then optimized crosssectional areas throughout this structure. Inefficient (low-area) members were then removed. Because the minimum value for I-beam cross-sectional area in the AISC manual is $0.914 \mathrm{in}^{2}$, this value was chosen as the threshold for removing members. The removed members were eliminated from the design variable vector (i.e., they do not reappear in the design), and the resulting topology was then optimized again. This process was continued until convergence was achieved. Convergence was defined as a loss of material less than $0.01 \%$ after member removal. For the numerical problems considered in this paper, convergence was achieved with at most three design iterations. It is noted that the ground structure is a statically indeterminate structure, and structural elements connecting two supports, which will be zero-force members, will cause stress singularity in determining the gradient [see Eq. (23)]. However, because these members do not contribute to transferring the external load, they were removed from the ground structure to avoid this singularity problem. Load magnitude and Young's modulus were scaled to 1 for all examples. The total amount of volume was set to $\nu L_{x} L_{y}$, where $L_{x}$ and $L_{y}$ are dimensions of the structure in the $x$ and $y$ directions, respectively. While the aim of stress-based design in the first and second example was to minimize the maximum of stress, the third example used both stress and compliance in the constraints while aiming to minimize the total volume of material [see Eq. (39)]. In all examples, the maximum was approximated with a $p$-norm function with an exponent of $p=100$.

\section{Cantilever Structure under a Lateral Load}

This section begins with the cantilever structure under a point load applied at a top corner as shown in Fig. 5. The frame width, height, and total allowable volume were $L, 2 L$, and $L^{2}$, respectively. A full ground structure was used, where each node was connected to all other nodes to provide more design freedom with an enlarged solution space.

Fig. 6 shows optimized topologies for the three selected percentiles using compliance-based (left) and the proposed stress-based algorithms (right). In this figure, line thicknesses signify relative member cross-sectional areas. All line thicknesses were normalized to the maximum of cross-sectional area among all final designs (which is the lowest outer column in the top right design). Comparing results of each row reveals that compliance-based designs featured a primary load path with members taking a considerable share of the available volume. This load path was supported with a few other members. However, stress-based topologies are generally more complex, featuring many load paths to mitigate the effect of stress concentration in primary members. Moreover, while in the first column the optimizer was allowed to choose members with a relatively low bending efficiency (from the 10th percentile of moment of inertia), the minimum compliance design transfered the load primarily with bending mechanisms, but the stress-based design load paths were more diversified and the optimizer used mainly axial load transfer mechanisms.

The number of elements in these final designs is shown in Table 1, where it can be seen that stress-based designs featured more members. Note that while the number of members decreased with choosing a higher percentile in the compliance-based

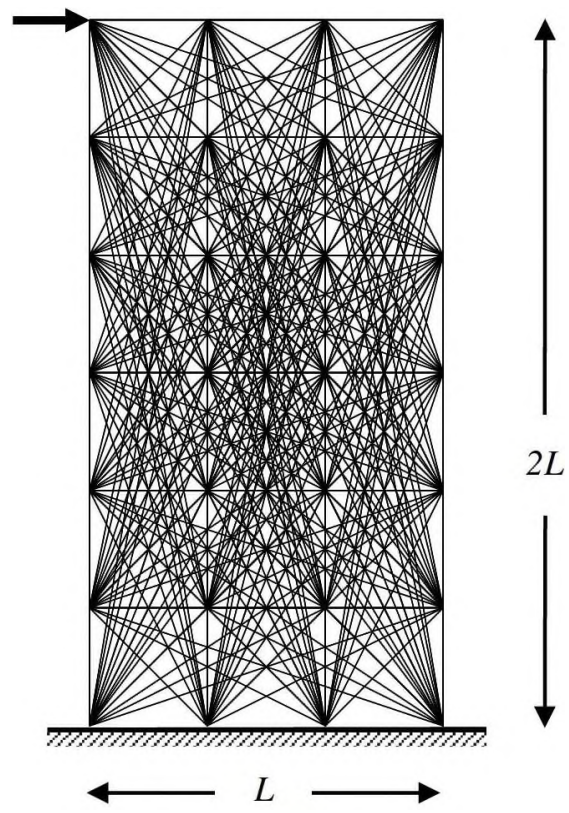

Fig. 5. Ground structure for the cantilever structure under a lateral load 


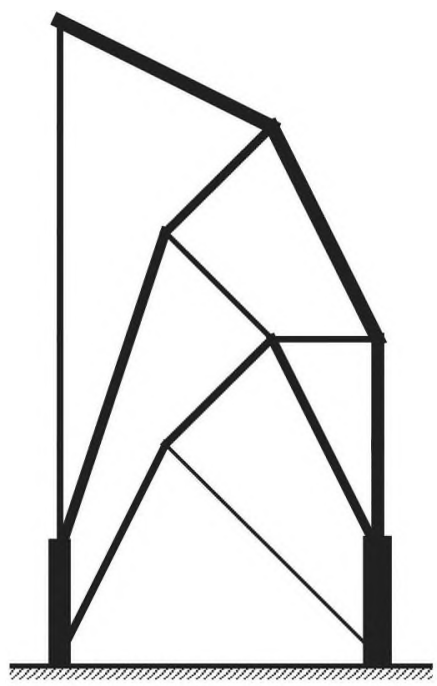

(a)

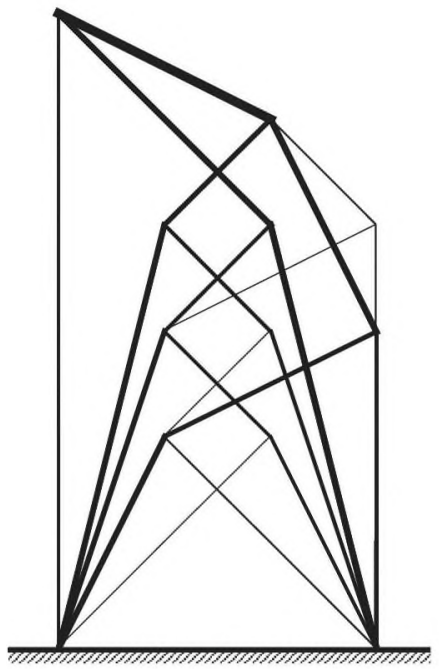

(d)

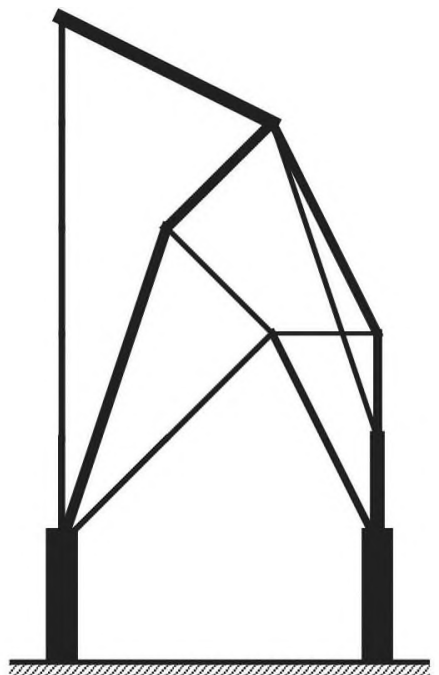

(b)

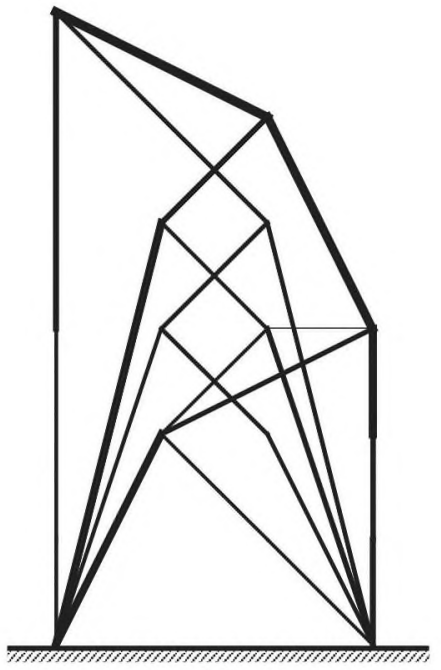

(e)

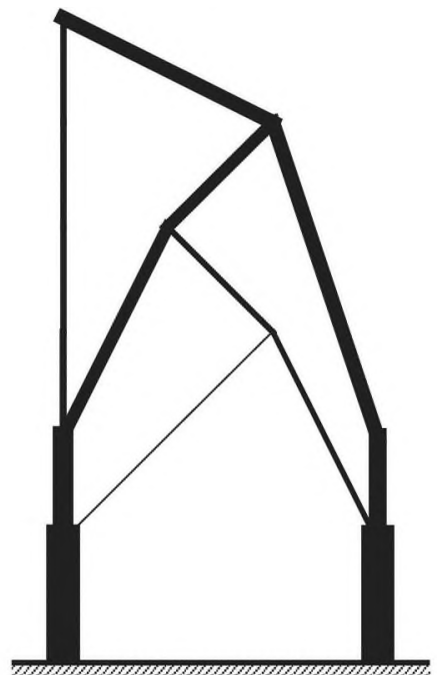

(c)

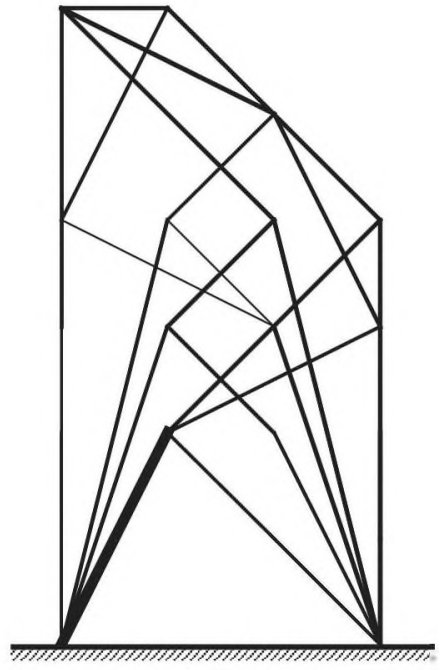

(f)

Fig. 6. Designs resulted from compliance-based (a-c) and stress-based (d-f) topology optimization of the cantilever structure for 10th, 50th, and 90th percentiles (from left to right) of cross-sectional moment of inertia

methodology (the dominant bending deflections were resisted with fewer members with larger cross-sectional areas), this was not the case for stress-based designs.

These differences in the designs point to a different design approach for stress-based design: avoiding large von Mises stresses in members instead of maximizing stiffness under the applied load, which is the target of compliance-based design. These differences are numerically investigated in Table 2 . It is observed that compliancebased design always resulted in a lower compliance (higher stiffness), but was outperformed by the stress-based design in terms of maximum of stress $\left(s_{\mathrm{max}}\right)$, average stress $\left(s_{\mathrm{ave}}\right)$. and standard deviation

Table 1. Number of Elements in the Compliance-Based and Stress-Based Designs for the Cantilever Structure

\begin{tabular}{lcc}
\hline Percentile & Compliance-based design & Stress-based design \\
\hline $10 \mathrm{th}$ & 20 & 37 \\
$50 \mathrm{th}$ & 19 & 29 \\
$90 \mathrm{th}$ & 16 & 38 \\
\hline
\end{tabular}

of stress $(\operatorname{std}[s])$. Comparing the designs columnwise (higher percentile for bending efficiency) shows that increasing the bending efficiency of members led to a consistent decrease in compliance (deflection at the point at which load is applied). However, maximum of stress increased. This might be because in a structure with more bending capacity, larger internal shear forces are also developed within the members, which leads to a greater von Mises criterion at every point of the structure; hence a higher maximum for the whole structure. Overall, axial load transfer mechanism appears to be

Table 2. Comparison of Design Performance for Designs of the for the Cantilever Structure

\begin{tabular}{lccclc}
\hline Percentile & Design & $C$ & \multicolumn{1}{c}{$S_{\max }$} & \multicolumn{1}{c}{$S_{\text {ave }}$} & std $[S]$ \\
\hline \multirow{2}{*}{ 10th } & Compliance-based & 54.934 & 0.122 & 0.074 & 0.017 \\
& Stress-based & 58.031 & 0.073 & 0.07 & 0.002 \\
50th & Compliance-based & 50.833 & 0.122 & 0.076 & 0.016 \\
& Stress-based & 59.363 & 0.079 & 0.075 & 0.005 \\
90th & Compliance-based & 47.035 & 0.112 & 0.079 & 0.017 \\
& Stress-based & 59.34 & 0.082 & 0.078 & 0.008 \\
\hline
\end{tabular}




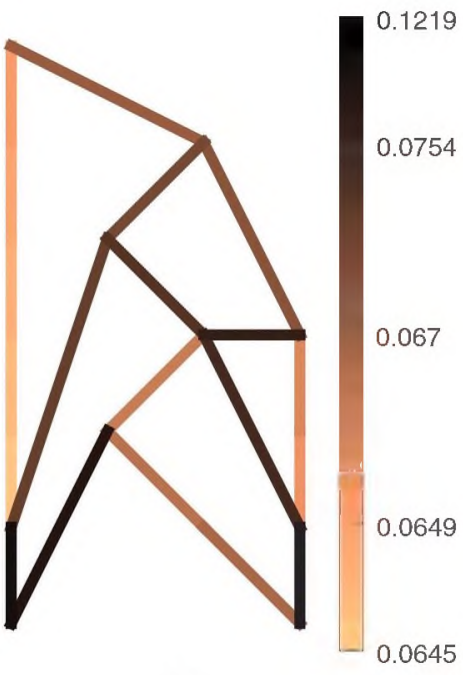

(a)

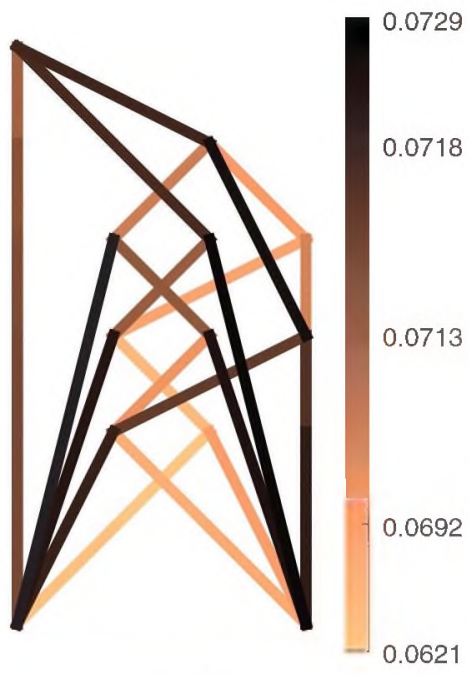

(d)

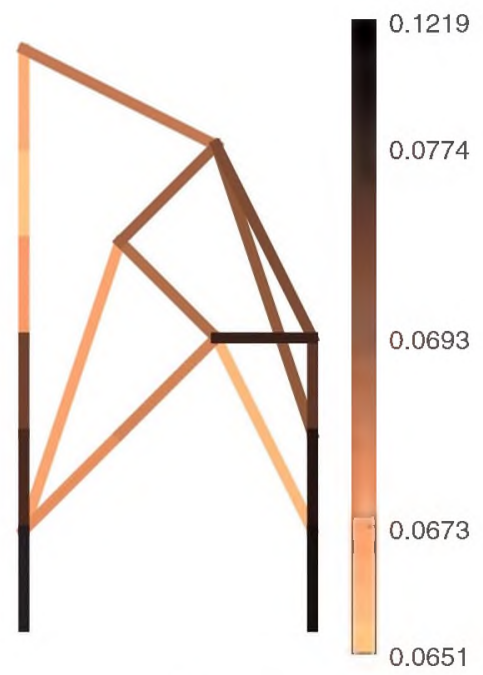

(b)

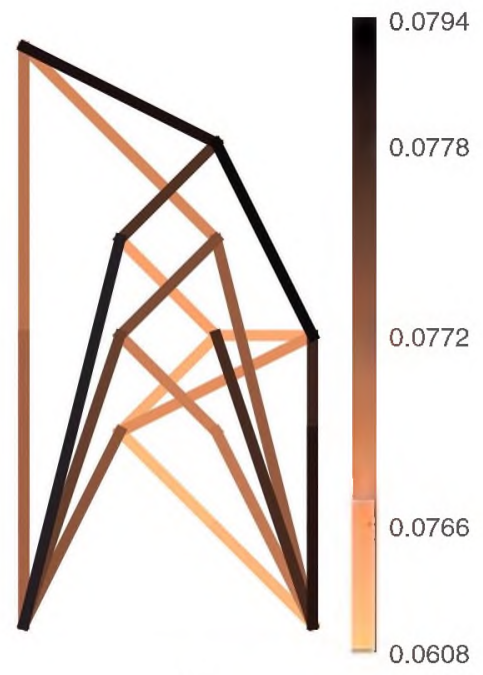

(e)

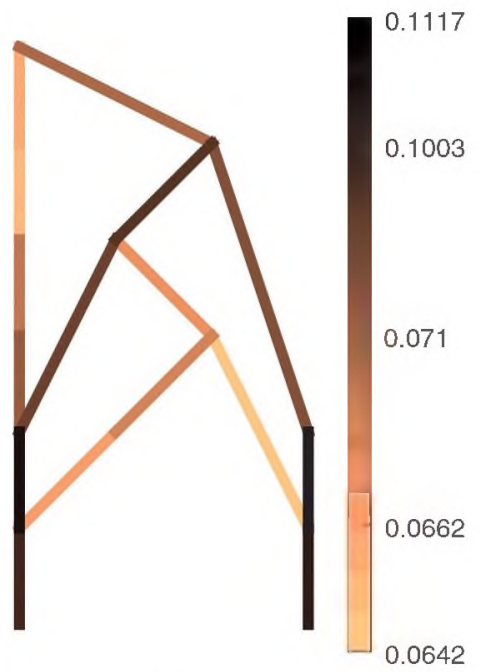

(c)

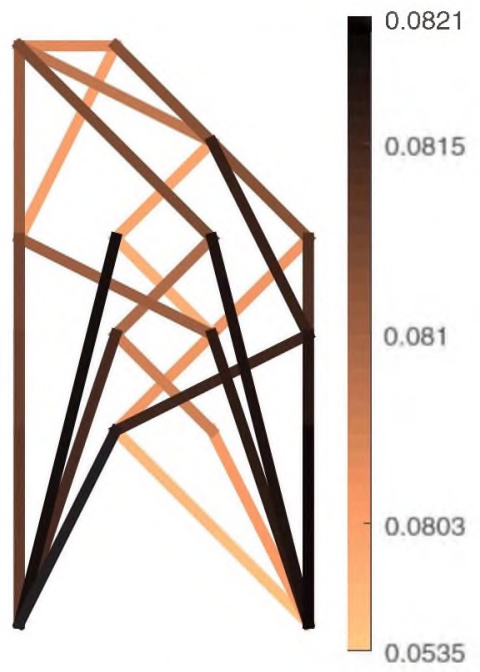

(f)

Fig. 7. Stress distribution for the designs shown in Fig. 6; intensity shows the magnitude for maximum of stress in each element

pursued by the optimizer with the proposed stress-based design objective.

Stress variations within the final designs are also depicted in Fig. 7. In this figure the maximum of stress in each member is chosen as the stress magnitude of that member. It can be seen that designs with the proposed methodology had significantly lower maximum stress magnitude with lower stress dispersion (although stress variance was not directly controlled for). It is noted that the location for maximum stress for the compliance-based design was always in the outer columns, but the stress-based design used several diagonal members in these locations to mitigate this effect. This modification, in turn, led to a change in the location of maximum stress for these designs.

\section{Bridge Under Distributed Loads}

The ground structure for this example was a bridge under distributed loads as shown in Fig. 8. Frame width, height, and total allowable volume were $L / 3,2 L$, and $2 / 3 L^{2}$, respectively. In this example the final designs were found using a fixed 50th percentile for the moment of inertia (median of the data).
Fig. 9 shows the optimized topologies using the described compliance-based and stress-based methodologies. The compliancebased design devoted a significant share of volume to the middle of the bottom and the top chord members. These members gradually received less volume progressing from the center toward the supports. This pattern is similar to the previous compliance-based designs, where elements with large area were observed to control the deflections (mainly due to bending). The vertical link member in the center controlled the maximum deflection, which occured in the center. The stress-based design, on other hand, consisted of more

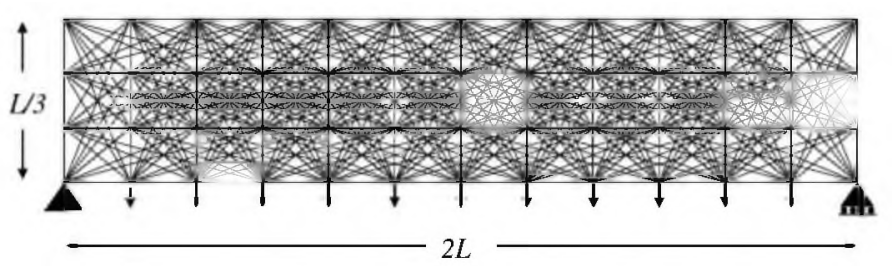

Fig. 8. Ground structure for the bridge structure under distributed load 


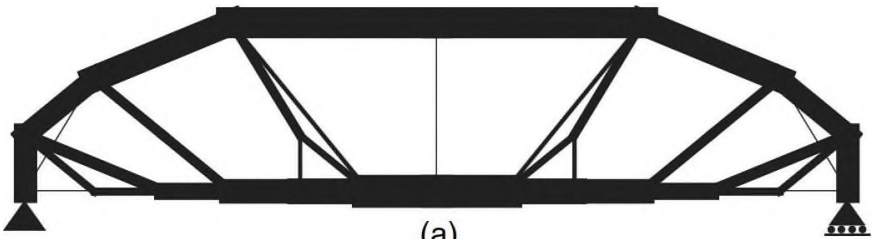

(a)

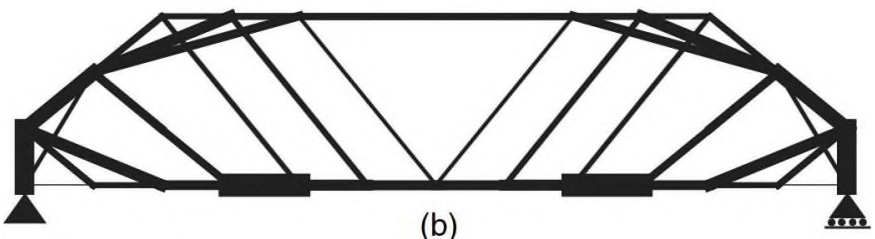

(b)

Fig. 9. Compliance-based and stress-based designs for the bridge structure

members with more even material distribution throughout the domain. Moreover, the topology resembled the stress variations within a simply supported continuum beam under distributed loads. Under this analogy, the diagonal members resisted the developed shear stresses and the top and bottom chords resisted bending stresses. These shear members were angled and were designed to be stronger closer to supports where shear was higher. The bottom chord in this design did not receive the highest share of volume in the center where bending was maximum. Instead, adjacent members that resist a combination of bending and shear stresses (which results in a higher von Mises stress) were designed to be stronger.

The von Mises stress distributions within these two designs are shown in Fig. 10, where it can be seen that the maximum stress in the compliance-based design (0.1349) exceeded that of the stressbased design (0.1143). Moreover, in the stress-based design the stresses in members adjacent to central members in the bottom chord were larger, which justifies their stronger design. In addition, the stress in diagonal members of this design increased toward the supports, which is consistent with the internal shear variations of the continuum beam analogy. Similar to the preceding example, less variability was observed in the stress distribution of the stress-based design. Finally, it is noted that the location of maximum stress was different in these two designs. For the compliancebased design the maximum stress was in the bottom chord where it met the support. However, these members were designed with relatively small cross-sectional areas, which makes this design more susceptible to failure due to stress.
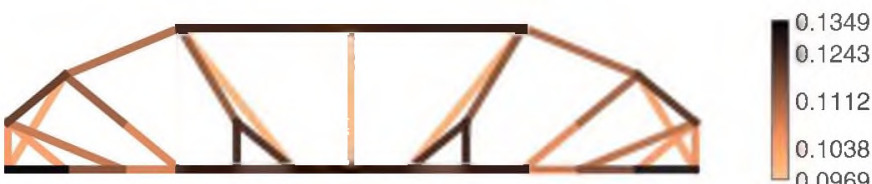

(a)
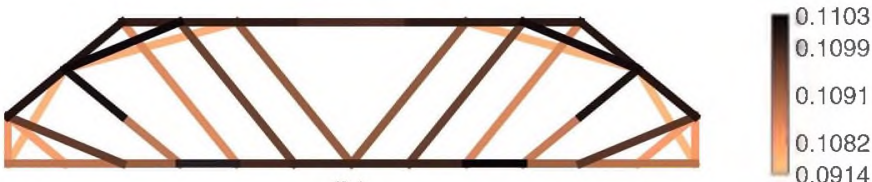

(b)

Fig. 10. Stress distribution for compliance-based and stress-based designs for the bridge structure
A methodology to interpret the designs resulted from the proposed methodology to actual I-beam sections from the AISC manual is now discussed. The proposed methodology uses a continuous relationship (motivated by the AISC data) for the design variables, and other cross-sectional properties. However, section properties are discrete numbers in the AISC (or any other) design manual. Therefore the final designs need to be mapped into actual cross-sections from the AISC I-beams database. This interpretation should be done so that the performance loss of the design is minimum. Therefore the methodology uses cross section closest to each designed member by the proposed algorithm as measured by the Euclidian distance in the six-dimensional coordinates of member area, moment of inertia, cross-sectional height, flange width, flange thickness, and web thickness. However, because moment of inertia is significantly larger than the other section properties, its square root was used in determining the distance. As an example, this methodology was used to interpret the compliance-based and stress-based bridge designs as actual cross sections from the AISC design manual. The results are shown in Appendix II, where the change in performance is less than $3 \%$.

\section{Cantilever Structure under Central Load}

The preceding examples showed how directly controlling for stress impacts the final design in representative percentiles of member cross-sectional bending efficiency. Moreover, the stress-based designs were compared with compliance-based designs. In this example, effects of controlling both stress and compliance on the volume of material used in the final design is investigated. The design statement is expressed as follows:

$$
\min _{\mathbf{a}} v=\mathbf{a}^{T} \mathbf{l}
$$

$$
\text { s.t. } \quad \mathbf{K}(\mathbf{a}) \mathbf{d}(\mathbf{a})=\mathbf{f}
$$

$\mathbf{f}^{T} \mathbf{d}(\mathbf{a}) \leq \gamma C^{*}$

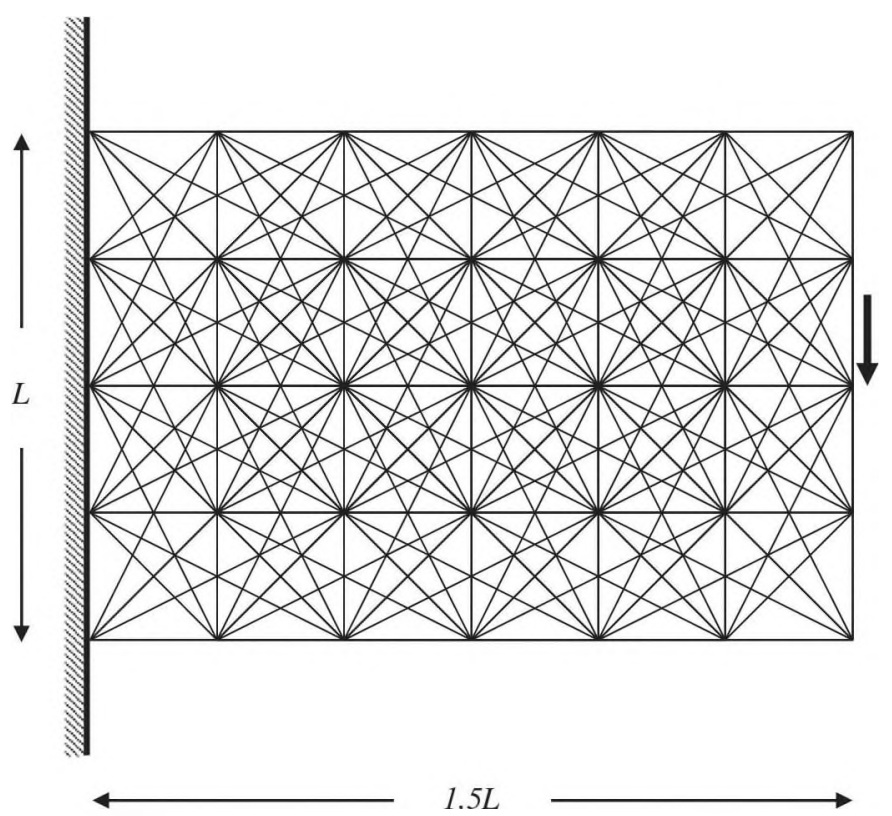

Fig. 11. Ground structure for horizontal cantilever structure under central load 


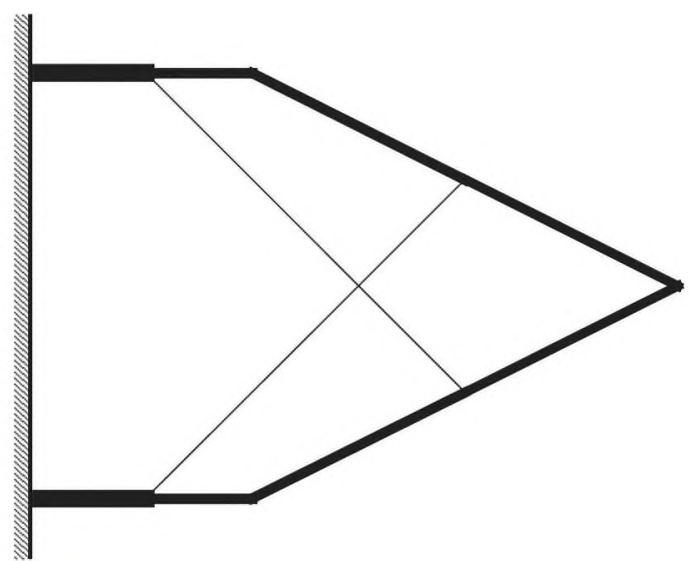

(a)
$N_{e}=12$

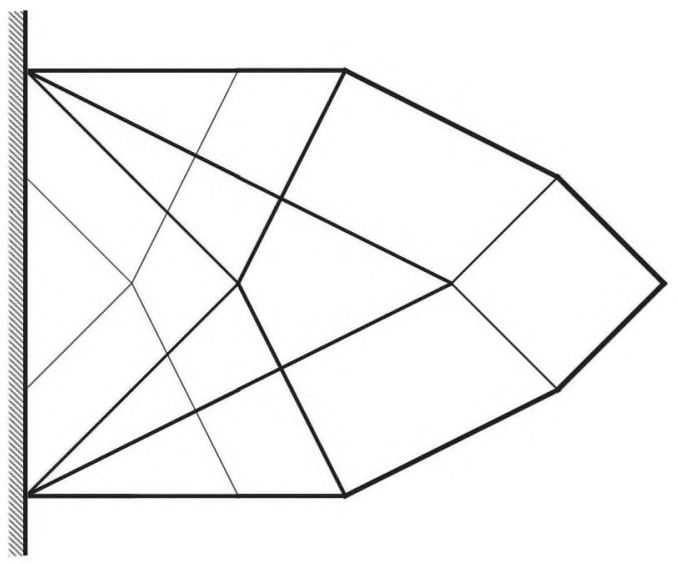

(b)

$N_{e}=34$

Fig. 12. Compliance-based and stress-based designs for the horizontal cantilever structure: (a) compliance-based design ( $N_{e}=12$ ); (b) stress-based design $\left(N_{e}=34\right)$

$$
\begin{gathered}
\left(\sum_{a=1}^{m} \sum_{\beta=1}^{n} S_{\alpha \beta}^{p}\right)^{1 / p} \leq \lambda S^{*} \\
a_{\min }<a \leq a_{\max }
\end{gathered}
$$

where $C^{*}$ and $S^{*}=$ allowable compliance and stress (capacities), respectively; and $\gamma$ and $\lambda=$ coefficients (designer's choice) for importance of either stress or compliance (as a proxy for stiffness), respectively, in the final design. Note that this problem statement features three nonlinear constraints, which increase the computational time. Therefore a ground structure with partial connectivity (i.e., not fully connected but not with very simple connectivity) with 283 members was used for this example as a reasonable compromise to allow illustrating the effects of controlling both stress and compliance. This ground structure geometry, the boundary conditions, and the applied load are shown in Fig. 11. The width and height of this frame were equal to $L$ and $1.5 L$, respectively.

One way to obtain reasonable values for capacities $C^{*}$ and $S^{*}$ is to optimize this ground structure using design statements in Eqs. (1) and (20) separately under a limited volume of material. In practical situations, $C^{*}$ could be selected as a fraction of structure height and $S^{*}$ could be determined from material strength. Nevertheless,

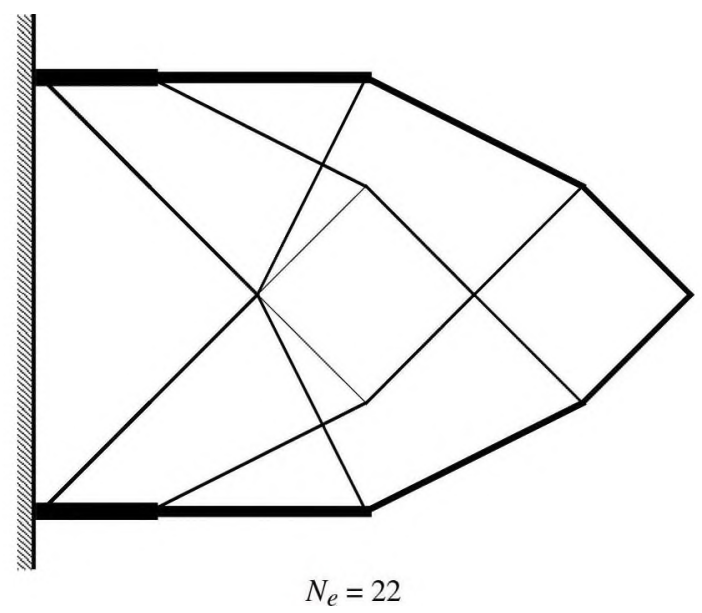

Fig. 13. Optimized topology using the minimum volume design statement in Eq. (39) with $\gamma=\lambda=1$ the first approach under a total volume of $1.5 L^{2}$ was used here. The results for these two limiting cases are shown in Fig. 12, where line thicknesses are normalized by the maximum area among all designs. As expected, differences between the two designs were similar to those observed in the preceding examples. In all the design that follow, $N_{e}$ shows the number of elements remaining in the final design.

The problem statement in Eq. (39), with a values of 1 for both $\gamma$ and $\lambda$, was used and this frame topology was designed using $C^{*}$, and $S^{*}$ from the preceding limiting cases. The resulting design is shown in Fig. 13. As member thicknesses indicate, this design required approximately $12 \%$ more material compared with the two limiting cases shown in Fig. 12. Moreover, features of both of the preceding topologies can be observed because the design was required to satisfy both stress and compliance constraints.

The effects of moving the design toward each limiting state of stress or compliance were investigated by changing the coefficients of $\lambda$ and $\gamma$ in Eq. (39). Designs for several combinations are presented in Fig. 14. Identical normalization of member thickness was used across all these designs. The designs in the top row were based on a tighter control over compliance (left design, with $\gamma=0.5$ ) and over stress (right design, with $\lambda=0.5$ ), and the designs on the bottom row were for relaxed constraints ( $\lambda=2$ or $\gamma=2$ ). Comparing these two rows, it is observed that, as expected, designs for tighter control over stress or compliance required more volume (approximately twice as much). The most complex design was achieved when the highest control over stress was requested, which was significantly different compared with all other designs. Finally, the design for $\gamma=1$ and $\lambda=2$, although it required less volume, tended toward the compliance-based design, and for $\gamma=2$ and $\lambda=1$ was similar to the design shown in Fig. 13 .

\section{Concluding Remarks}

This paper proposed methodologies to advance frame gradientbased topology optimization on two fronts. First, actual data for standard factory-built sections were used in the structural design. Quantile regression was used to express moment of inertia and other cross-sectional properties analytically as functions of member cross-sectional area, and to account for the relatively large variance in the data set. Optimized designs were found using several percentiles of cross-sectional bending efficiency. Although 


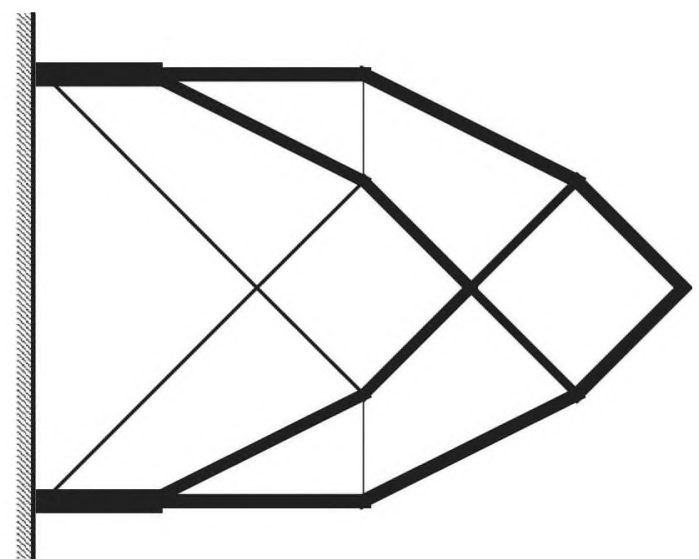

(a)

$N_{e}=20$

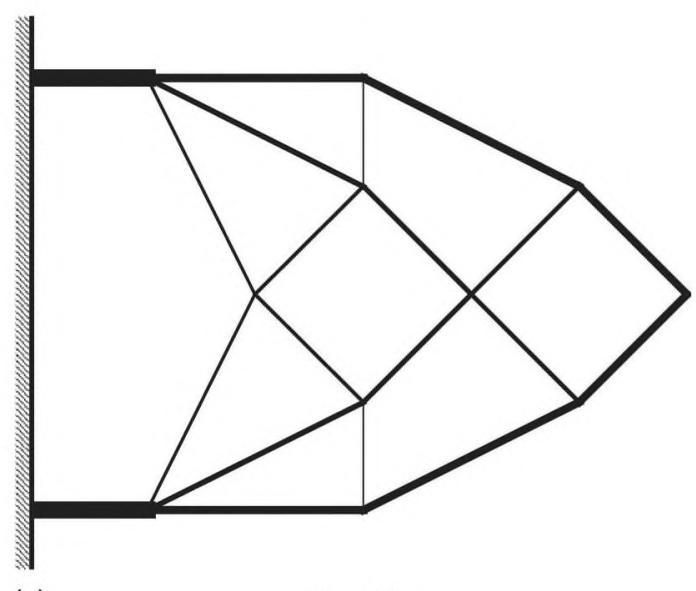

(c)
$N_{e}=20$

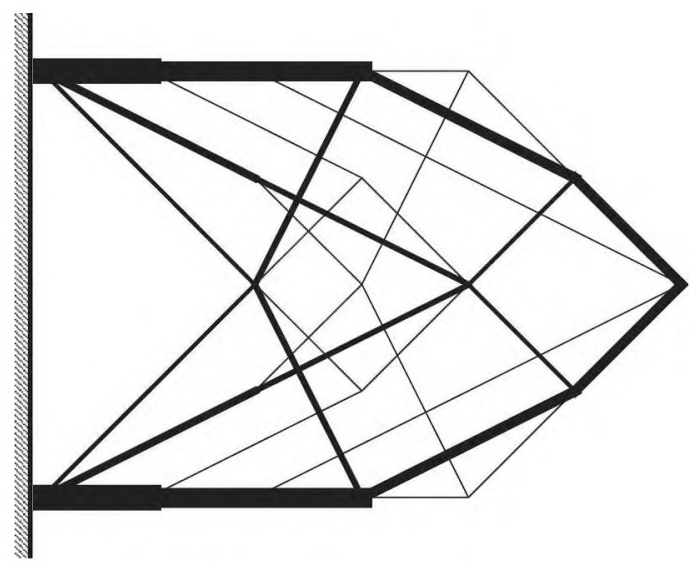

(b)

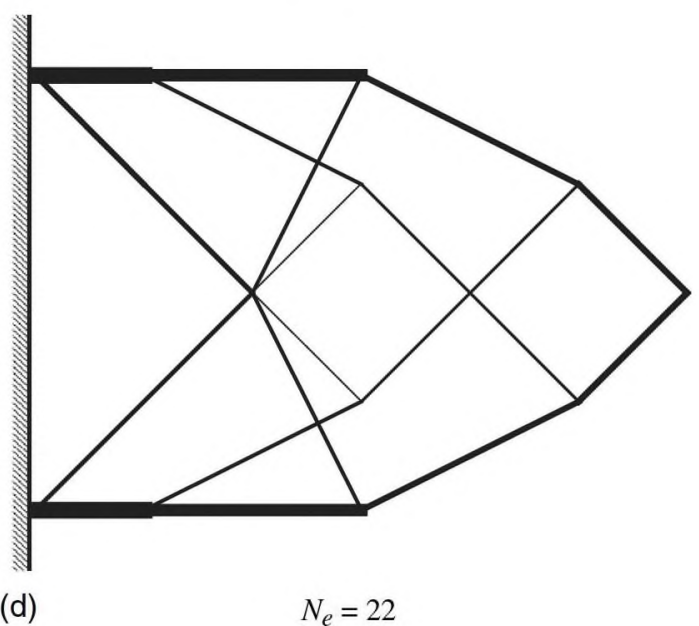

Fig. 14. Minimum volume designs under several compliance and stress constraints: (top) tight constraints; (bottom) relaxed constraints: (a) $\gamma=0.5$, $\lambda=1, N_{e}=20 ;$ (b) $\gamma=1, \lambda=0.5, N_{e}=42$; (c) $\gamma=1, \lambda=2, N_{e}=20$; (d) $\gamma=2, \lambda=1, N_{e}=22$

the examples used a single percentile for all members in each design, the algorithm could be implemented with flexibility to use different percentiles for different members. Second, an algorithm for stress-based topology optimization using the von Mises yield criterion was proposed to directly control the maximum of stresses in frames. A comparison of stress-based and the widely used compliance-based designs was made, and it was observed that stress-based designs generally feature load path diversification to reduce stress in members. Stress distribution within the final designs verified the effectiveness of the proposed algorithm. Additionally, combined effects of controlling for compliance and stress on the final design were investigated.

The free-from capability of topology optimization is best suited for initial stages of design conceptualization. However, the resulting topologies depend on, among others, the defined objective functions (stress-based or compliance-based) and crosssectional properties of frame members. Therefore methodologies to directly incorporate these within the design procedure are needed. The proposed algorithm achieves these with computational efficiency. In addition, a methodology to interpret these designs in terms of the AISC manual section was proposed, which makes this algorithm attractive for a systematic search of the design space for optimized connectivity and relative sizing of members. These designs can then be investigated more thoroughly for satisfying other engineering and architectural constraints. A limitation of this work is that buckling (overall and individual member) constraints were not included. Including these is the subject of future research.

\section{Appendix I. Equations of Fitted Curves Given by Quantile Regression}

This appendix provides quantile regression results for expressing cross-sectional properties in terms of area for the I-beams in AISC manual. These results are obtained using the MATLAB code developed by Grinsted (2015). As discussed previously, these equations are needed to analytically derive the sensitivity of objective function or constraints. This section begins with results for $10 \%$

$$
\begin{gathered}
I=\left(1.0986 a-5.2573 \times 10^{-3} a^{2}+1.2719 \times 10^{-5} a^{3}\right)^{2} \\
h=3.0527 \sqrt{a}-1.7854 \times 10^{-1} a+4.8912 \times 10^{-3} \sqrt[3]{a^{3}} \\
b_{f}=1.6789 \sqrt{a}-1.9932 \times 10^{-2} a-1.3470 \times 10^{-3} \sqrt[2]{a^{3}} \\
t_{f}=1.2143 \times 10^{-1} \sqrt{a}-4.2559 \times 10^{-3} a+1.0328 \times 10^{-3} \sqrt[2]{a^{3}} \\
t_{w}=1.0601 \times 10^{-1} \sqrt{a}-7.2455 \times 10^{-3} a+7.1125 \times 10^{-4} \sqrt[3]{a^{3}}
\end{gathered}
$$



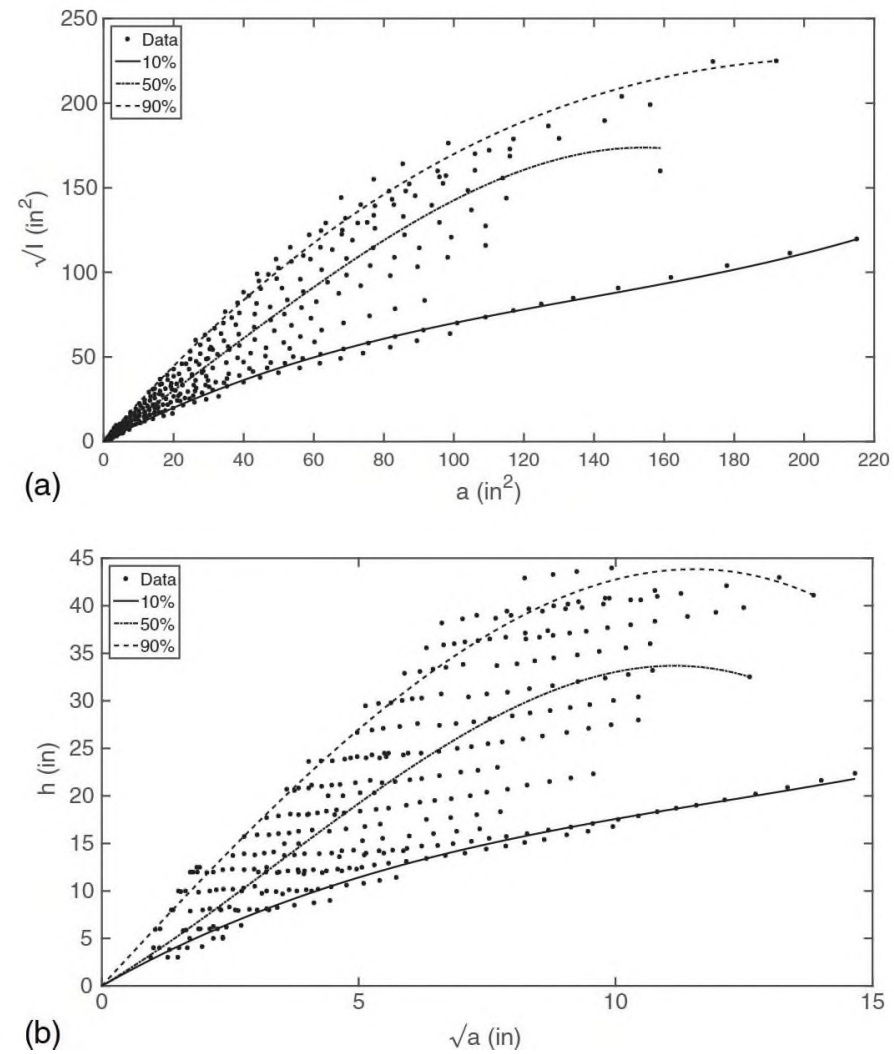

Fig. 15. Fitted curves given by quantile regression for predicting section properties overlaid on the transformed data: (a) moment of inertia; (b) section depth

For $50 \%$ the following are obtained:

$$
\begin{gathered}
I=\left(1.4614 a+2.9924 \times 10^{-3} a^{2}-3.3481 \times 10^{-5} a^{3}\right)^{2} \\
h=3.2538 \sqrt{a}+2.2934 \times 10^{-1} a-2.2441 \times 10^{-2} \sqrt[3]{a^{3}} \\
b_{f}=2.2517 \sqrt{a}-5.4645 \times 10^{-2}-1.2316 \times 10^{-3} \sqrt[2]{a^{3}} \\
t_{f}=1.6280 \times 10^{-1} \sqrt{a}-8.3381 \times 10^{-3} a+1.3689 \times 10^{-3} \sqrt[2]{a^{3}}
\end{gathered}
$$

$$
t_{w}=1.1882 \times 10^{-1} \sqrt{a}-8.8533 \times 10^{-3} a+9.2161 \times 10^{-4} \sqrt[2]{a^{3}}
$$

Finally for $90 \%$, the results are

$$
\begin{aligned}
& I=\left(2.3711 a-7.2319 \times 10^{-3} a^{2}+5.1265 \times 10^{-6} a^{3}\right)^{2} \\
& h=5.8712 \sqrt{a}-2.9532 \times 10^{-2} a-1.2999 \times 10^{-2} \sqrt[2]{a^{3}} \\
& h_{f}=3.6792 \sqrt{a}-2.5630 \times 10^{-1} a+5.9855 \times 10^{-3} \sqrt[2]{a^{3}} \\
& t_{f}=1.6174 \times 10^{-1} \sqrt{a}+3.2577 \times 10^{-3} a+5.9890 \times 10^{-1} \sqrt[2]{a^{3}} \\
& t_{w}=1.3544 \times 10^{-1} \sqrt{a}-3.9493 \times 10^{-3} a+6.2998 \times 10^{-4} \sqrt[2]{a^{3}}
\end{aligned}
$$

Fitted curves over the actual data are shown in Fig. 15.

\section{Appendix II. Results of Interpreting Final Designs of Bridge Structure to AISC I-Beam Sections}

This section demonstrates the bridge stress-based design interpreted in terms of the AISC design manual.

First, an element numbering was defined as shown in Fig. 16. Next, the methodology proposed in the Numerical Examples section was used for selecting the closest actual AISC cross sections. The resulting cross sections along with predicted cross-sectional properties are shown in Table 3. For this specific example, the maximum stress of $S_{\max }=0.1135$ was obtained for the interpreted design, which resulted in less than a $1 \%$ change in stress-based performance. Similarly, all other resulting designs were interpreted as the AISC cross sections. This resulted in a change of compliance of the compliance-based design for the bridge structure of approximately $2 \%$. The change in volume for both bridge designs was less than $2 \%$ (volume loss). Moreover, a maximum change in performance of less than 9\% was observed among all other designs (stress-based or compliance-based). These results are not shown here for brevity. This gap could be reduced to approximately $1 \%$ if all resulting areas were increased by $10 \%$ before interpreting them as the AISC sections. However, it is noted that this approach increases the volume of material assumed by the proposed algorithm during the design procedure.

Finally, the predicted values for cross-sectional properties by the proposed methodology were verified. Table 3 shows that some AISC cross sections were used more than once. Two sections that were used most frequently were selected (W16 $\times 77$ and $\mathrm{W} 12 \times 45$ ), and their cross-sectional properties were obtained using the AISC manual. The averages of the predicted values among the three members were taken, and the percentage errors between these averages and the actual AISC values are presented in Table 4.

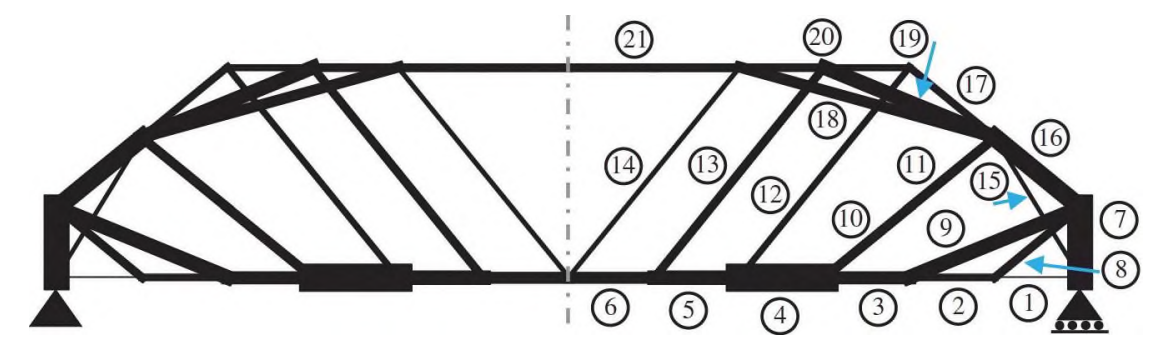

Fig. 16. Element numbering for interpreting the stress-based bridge design in terms of the AISC design manual 
Table 3. Interpreted Sections from the AISC Manual for the Bridge Stress-Based Design, and Predicted Cross Section Properties from the Proposed Algorithm

\begin{tabular}{|c|c|c|c|c|c|c|c|}
\hline \multirow{2}{*}{$\begin{array}{l}\text { Element } \\
\text { number }^{\mathrm{d}}\end{array}$} & \multirow{2}{*}{$\begin{array}{l}\text { AISC equivalent } \\
\text { section }\end{array}$} & \multicolumn{6}{|c|}{ Predicted values } \\
\hline & & $a$ & $I$ & $h$ & $b_{f}$ & $t_{f}$ & $t_{w}$ \\
\hline 1 & W6 $\times 16$ & 3.6402 & 28.7 & 6.8870 & 4.0887 & 0.2898 & 0.2009 \\
\hline 2 & $\mathrm{~W} 12 \times 45$ & 11.8604 & 313.2 & 13.0091 & 7.0563 & 0.5177 & 0.3419 \\
\hline 3 & $\mathrm{~W} 16 \times 77$ & 21.8468 & $1,089.4$ & 17.9271 & 9.2052 & 0.7186 & 0.4561 \\
\hline 4 & W24 × 207 & 53.7205 & $6,716.1$ & 27.3326 & 13.0835 & 1.2843 & 0.7582 \\
\hline 5 & W16 x 89 & 24.6149 & $1,390.2$ & 19.0477 & 9.6761 & 0.7696 & 0.4842 \\
\hline 6 & W $16 \times 77$ & 20.7622 & 981.7 & 17.4645 & 9.0091 & 0.6982 & 0.4448 \\
\hline 7 & W24 × 162 & 45.5711 & $4,850.1$ & 25.5127 & 12.3315 & 1.1402 & 0.6822 \\
\hline 8 & $\mathrm{~W} 14 \times 53$ & 15.315 & 527.3 & 14.9008 & 7.9013 & 0.5915 & 0.3847 \\
\hline 9 & $\mathrm{~W} 16 \times 100$ & 25.9493 & $1,548.6$ & 19.5597 & 9.8897 & 0.7939 & 0.4974 \\
\hline 10 & W14 × 61 & 17.2158 & 669.5 & 15.8458 & 8.3141 & 0.6297 & 0.4064 \\
\hline 11 & W14 × 68 & 17.9637 & 730.3 & 16.2018 & 8.4683 & 0.6444 & 0.4148 \\
\hline 12 & $\mathrm{~W} 12 \times 40$ & 11.1516 & 276.3 & 12.5875 & 6.8642 & 0.5016 & 0.3324 \\
\hline 13 & $\mathrm{~W} 12 \times 45$ & 12.2855 & 336.5 & 13.2559 & 7.1681 & 0.5271 & 0.3474 \\
\hline 14 & $\mathrm{~W} 10 \times 22$ & 6.5366 & 93.5 & 9.4429 & 5.3792 & 0.3846 & 0.2613 \\
\hline 15 & $\mathrm{~W} 10 \times 26$ & 7.4442 & 121.7 & 10.1291 & 5.7118 & 0.4099 & 0.2770 \\
\hline 16 & $\mathrm{~W} 18 \times 130$ & 32.7215 & 2,485 & 21.9164 & 10.862 & 0.9147 & 0.5625 \\
\hline 17 & $\mathrm{~W} 12 \times 50$ & 13.5234 & 409.1 & 13.9509 & 7.4803 & 0.5540 & 0.3631 \\
\hline 18 & $\mathrm{~W} 16 \times 77$ & 20.2847 & 936.1 & 17.2564 & 8.9205 & 0.6892 & 0.4398 \\
\hline 19 & $\mathrm{~W} 18 \times 97$ & 26.0924 & $1,566.1$ & 19.6135 & 9.9121 & 0.7965 & 0.4988 \\
\hline 20 & $\mathrm{~W} 12 \times 40$ & 11.3687 & 287.4 & 12.7180 & 6.9238 & 0.5066 & 0.3353 \\
\hline 21 & $\mathrm{~W} 12 \times 45$ & 12.6251 & 355.7 & 13.4500 & 7.2557 & 0.5346 & 0.3518 \\
\hline
\end{tabular}

${ }^{\mathrm{a}}$ See Fig. 16 for element numbering.

Table 4. Predicted Section Properties of Two Most Frequently Used Equivalent AISC Sections in the Interpreted Bridge Design

\begin{tabular}{|c|c|c|c|c|c|c|c|}
\hline Element number & AISC equivalent section & $a$ & $I$ & $h$ & $b_{f}$ & $t_{f}$ & $I_{w}$ \\
\hline & W $16 \times 77$ & 22.6 & 1110 & 16.5 & 10.3 & 0.760 & 0.455 \\
\hline \multicolumn{8}{|l|}{$3,6,18$} \\
\hline & Average of prediction & 20.9646 & $1,002.4$ & 17.5493 & 9.0449 & 0.7020 & 0.4469 \\
\hline & Error \% & 7.2363 & 9.6937 & -6.3594 & 12.1854 & 7.6316 & 1.7802 \\
\hline & W $12 \times 45$ & 13.1 & 348 & 12.1 & 8.05 & 0.575 & 0.335 \\
\hline \multicolumn{8}{|l|}{$2,13,21$} \\
\hline & Average of prediction & 12.2570 & 335.1333 & 13.2383 & 7.1600 & 0.5265 & 0.3470 \\
\hline & Error $\%$ & 6.4351 & 3.6973 & -9.4074 & 11.0559 & 8.4348 & -3.5821 \\
\hline
\end{tabular}

In general, the prediction errors seem acceptable. The maximum error seems to be in predicting $b_{f}$. It is again noted that the interpreted design performance has changed by less than $1 \%$.

\section{References}

Achtziger, W. (1999). "Local stability of trusses in the context of topology optimization. Part I: Exact modelling." Struct. Optim., 17(4), 235-246.

Achtziger, W., and Stolpe, M. (2007). "Truss topology optimization with discrete design variables guaranteed global optimality and benchmark examples." Struct. Multidiscip. Optim., 34(1), 1-20.

AISC (American Institute of Steel Construction). (2015). "Steel construction manual shapes database." (https://www.aisc.org/globalassets /product-files-not-searched/manuals/aiscshapesdatabasev14_1-current.zip〉 (Feb. 12, 2017).

Amini, K., Jalalpour, M., and Delatte, N. (2016). "Advancing concrete strength prediction using non-destructive testing: Development and verification of a generalizable model." Constr. Build. Mater. 102(1), $762-768$.

Beghini, L. L., Beghini, A.. Baker, W. F., and Paulino, G. H. (2014). "Integrated discrete/continuum topology optimization framework for stiffness or global stability of high-rise buildings." J. Struct. Eng., 141(8), 04014207.

Bendsoe, M. P., and Sigmund, O. (2004). Topology optimization: Theory, methods and applications, Springer, Stone Harbor, NJ.
Bruggi, M. (2008). "On an alternative approach to stress constraints relaxation in topology optimization." Struct. Multidiscip. Optim., 36(2), $125-141$.

Changizi, N., and Jalalpour, M. (2017). "Robust topology optimization of frames under geometric or material uncertainty." Struct. Multidiscip. Optim., 398-407.

Changizi, N., Kaboodanian, H., and Jalalpour, M. (2017). "Stress-based topology optimization of frame structures under geometric uncertainty." Comput. Methods Appl. Mech. Eng., 315, 121-140.

Cheng. G., and Guo, X. (1997). " $\varepsilon$-relaxed approach in structural topology optimization." Struct. Optim., 13(4), 258-266.

Cheng, G., and Jiang, Z. (1992). "Study on topology optimization with stress constraints." Eng. Optim., 20(2), 129-148.

Duysinx, P., and Bendsøe, M. P. (1998). "Topology optimization of continuum structures with local stress constraints." Int. J. Numer. Methods Eng., 43(8), 1453-1478.

Duysinx, P., and Sigmund, O. (1998). "New developments in handling stress constraints in optimal material distribution." Proc.,7th AIAA/ USAF/NASAISSMO Symp. on Multidisciplinary Analysis and Optimization, AIAA, Reston, VA, Vol. 1, 1501-1509.

Fredricson, H. (2005). "Topology optimization of frame structuresjoint penalty and material selection." Struct. Multidiscip. Optim., 30(3), $193-200$.

Fredricson, H., Johansen, T., Klarbring, A., and Petersson, J. (2003). "Topology optimization of frame structures with flexible joints." Struct. Multidiscip. Optim., 25(3), 199-214. 
Gaynor, A. T., Guest, J. K., and Moen, C. D. (2012). "Reinforced concrete force visualization and design using bilinear truss-continuum topology optimization.” J. Struct. Eng., 10.1061/(ASCE)ST.1943-54IX.0000692, 607-618.

Grinsted, A. (2015). "Quantile regression." 〈http://www.mathworks.com /matlabcentral/fileexchange/321 15-quantreg-X-y-tau-order-nboot-) (Jul. 7 , 2016).

Kanno, Y., and Guo, X. (2010). "A mixed integer programming for robust truss topology optimization with stress constraints." Int. J. Numer. Methods Eng., 83(13), 1675-1699.

Kirsch, U. (1990). "On singular topologies in optimum structural design." Struct. Optim., 2(3), 133-142.

Koenker, R., and Bassett, G., Jr. (1978). "Regression quantiles." Econometrica: J. Econometric Soc., 46(1), 33-50.

Kureta, R., and Kanno, Y. (2014). "A mixed integer programming approach to designing periodic frame structures with negative poissons ratio." Optim. Eng., 15(3), 773-800.

Le, C., Norato, J., Bruns, T., Ha, C., and Tortorelli, D. (2010). "Stress-based topology optimization for continua." Struct. Multidiscip. Optim., 41(4), 605-620.

Liang, Q. Q., Xie, Y. M., and Steven, G. P. (2000). "Optimal topology design of bracing systems for multistory steel frames." J. Struct. Eng., 10.1061/(ASCE)0733-9445(2000)126:7(823), 823-829.

MATLAB vR2016a [Computer software]. MathWorks, Natick, MA.

Mogami, K., Nishiwaki, S., Izui, K., Yoshimura, M., and Kogiso, N. (2006). "Reliability-based structural optimization of frame structures for multiple failure criteria using topology optimization techniques." Struct. Multi. Optim., 32(4), 299-311.

Ohsaki, M., and Katoh, N. (2005). "Topology optimization of trusses with stress and local constraints on nodal stability and member intersection." Struct. Multidiscip. Optim., 29(3), 190-197.

Pedersen, C. B. (2003). "Topology optimization design of crushed 2D-frames for desired energy absorption history." Struct. Multidiscip. Optim., 25(5-6), 368-382.

Pedersen, C. B. (2004). "Crashworthiness design of transient frame structures using topology optimization." Comput. Methods Appl. Mech. Eng.. 193(6), 653-678.

Rasmussen, M., and Stolpe, M. (2008). "Global optimization of discrete truss topology design problems using a parallel cut-and-branch method." Comput. Struct., 86(13), 1527-1538.
Richardson, J. N., Adriaenssens, S., Bouillard, P., and Coelho, R. F. (2012) "Multiobjective topology optimization of truss structures with kinematic stability repair." Struct. Multidiscip. Optim., 46(4), 513-532.

Rojas-Labanda, S., and Stolpe, M. (2015). "Benchmarking optimization solvers for structural topology optimization." Struct. Multidiscip. Optim., 52(3), 527-547.

Rozvany, G. (2001). "On design-dependent constraints and singular topologies." Struct. Multidiscip. Optim., 21(2), 164-172.

Rozvany, G., and Sobieszczanski-Sobieski, J. (1992). "New optimality criteria methods: Forcing uniqueness of the adjoint strains by corner-rounding at constraint intersections." Struct. Optim., 4(3-4), $244-246$

Sarma, K. C., and Adeli, H. (2000). "Fuzzy genetic algorithm for optimization of steel structures." J. Struct. Eng., 10.1061/(ASCE)0733-9445 (2000) 126:5(596), 596-604

Shea, K., and Smith. I. F. (2006). "Improving full-scale transmission tower design through topology and shape optimization." J. Struct. Eng., 10.1061/(ASCE)0733-9445(2006)132:5(781), 781-790.

Sigmund, O. (2011). "On the usefulness of non-gradient approaches in topology optimization." Struct. Multidiscip. Optim., 43(5), 589-596.

Stolpe, M. (2015). "Truss topology optimization with discrete design variables by outer approximation." J. Global Optim., 61(1), $139-163$.

Svanberg, K. (1987). "The method of moving asymptotesa new method for structural optimization." Int. J. Numer. Methods Eng., 24(2), 359-373.

Swan, C. C., and Rahmatalla, S. (2001). "Does continuum topology optimization have a place in design of large-scale structures?" Structures 2001: A Structural Engineering Odyssey, AIAA, Reston, VA, $1-9$.

Takezawa, A., Nishiwaki, S., Izui, K., and Yoshimura, M. (2007). "Structural optimization based on topology optimization techniques using frame elements considering cross-sectional properties." Struct. Multidiscip. Optim., 34(1), 41-60.

Torii, A. J., Lopez, R. H., and Miguel, L. F. (2015). "Modeling of global and local stability in optimization of truss-like structures using frame elements." Struct. Multidiscip. Optim., 51(6), 1187-1198.

Yang, R., and Chen, C. (1996). "Stress-based topology optimization." Struct. Optim.. 12(2-3), 98-105. 